\title{
FLOW OF A DUSTY FLUID DUE TO WAVY MOTION OF A WALL FOR MODERATELY LARGE REYNOLDS NUMBERS
}

\author{
V. RAMAMURTHY and U.S. RAO \\ Department of Mathematics \\ Indian Institute of Technology \\ Kharagpur 721302, India \\ (Received January 22, 1987)
}

\begin{abstract}
The two-dimensional flow of a dusty fluid induced by sinusoidal wavy motion of an infinite wavy wall is considered for Reynolds numbers which are of magnitude greater than unity. While the velocity components of the fluid and the dust particles along the axial direction consist of a mean steady flow and a periodic flow, the transverse components of both the fluid and the dust consist only of a periodic flow. This is true both for the outer flow (the flow beyond the boundary layer) and the inner flow (boundary layer flow). It is found that the mean steady flow is proportional to the ratio $4 \pi^{2} a^{2} / L^{2}(a / L<<1)$, where $a$ and $L$ are the amplitude and the wavelength of the wavy wall, respectively. Graphs of the velocity components, both for the outer flow and the inner flow for various values of mass - concentration of the dust particles are drawn. It is found that the steady flow velocities of the fluid and the dust particles approach to a constant value. Certain interesting results regarding the axial and the transverse velocity components are also discussed.
\end{abstract}

\section{INTRODUCTION.}

The problems of flow of fluid induced by sinusoidal wavy motion of a wall have been discussed by Tanaka [1], Taylor [2] and others [3,4]. Tanaka discussed the problem both for small and moderately large Reynolds numbers. While discussing the problem for moderately large Reynolds numbers, he has shown that, if the thickness of the boundary layer is larger than the wave amplitude the technique employed for small Reynolds numbers can be applied to the case of moderately large Reynolds numbers also.

Recently while studying the flow of blood through mammalian capillaries, blood is taken to be a binary system of plasma (liquid phase) and blood cells (solid phase). In order to gain some insight into the peristaltic motion of blood in capillaries the authors are motivated to study the induced flow of a dusty or two-phase fluid by sinusoidal motion of a wavy wall.

In the present paper, the two dimensional flow of a dusty fluid for moderately large Reynolds numbers is studied on the basis of the boundary layer theory in the case where a thickness of the boundary layer is larger than the wave amplitude of the wall. We assume that the amplitude of the wavy wall is small but finite, so that the solutions are obtained interms of a series expansion with respect to the small amplitude. 
2. FORMULATION OF THE PROBLEM.

We consider a two-dimensional flow of an incompressible viscous dusty fluid due to an infinite sinusoidal wavy wall which executes progressing motion with constant speed. Taking the Cartesian coordinates with $x$-axis in the direction of the progression of the wave, and the y-axis perpendicular to it, the motion of the wall is described by

$$
y=h(x, t)=a \cos \frac{2 \pi}{L}(x-c t)
$$

where $a$ is the amplitude, $L$ the wavelength and $c$, the phase velocity of the wall. We assume that $(a / L)<<1$ so that $(2 \pi a / L)<1$.

The non-dimensional equations of motion of a dusty fluid as formulated by Saffman [6] are

$$
\begin{aligned}
& \vec{q}_{t}+(\vec{q} \cdot \operatorname{grad}) \vec{q}=-\operatorname{grad} p+\frac{1}{R} \nabla^{2} \vec{q}+\alpha \lambda\left(\vec{q}_{p}-\vec{q}\right) \\
& \vec{q}_{p t}+\left(\vec{q}_{p} \cdot \operatorname{grad}\right) \vec{q}_{p}=\alpha\left(\vec{q}-\vec{q}_{p}\right) \\
& \operatorname{div} \vec{q}_{p}=0 \\
& \operatorname{div} \vec{q}_{p}=0
\end{aligned}
$$

The boundary conditions are

$$
\begin{aligned}
& u=0, v=\frac{\partial h}{\partial t} \text { at } y=h(x, t), \\
& |u|,|v|,\left|u_{p}\right|,\left|v_{p}\right| \text { all }<\infty \text { as } y \rightarrow \infty
\end{aligned}
$$

where $h=\varepsilon \cos (x-t)$ and $\varepsilon=2 \pi a / L$. The equations (2.6) represent the no slip condition of the fluid on the wall, where an assumption has been made that the wall executes only transverse displacement at every point. The subscript $t$ denoting partial differentiation with respect to $t$, the characteristic length being $L / 2 \pi$, the characteristic time being $\mathrm{L} / 2 \pi \mathrm{c}$, the fluid velocity $\mathrm{q} \equiv(\mathrm{u}, \mathrm{v})$ and the particle velocity $q_{p} \equiv\left(u_{p}, v_{p}\right)$ being non-dimensionalised with characteristic speed $c$, the fluid pressure $p$ being non-dimensionalised with characteristic pressure $c^{2}$, the non-dimensional parameters being $\lambda=\mathrm{mN}_{0} / \rho, \alpha=\mathrm{KL} / 2 \pi \mathrm{cm}, \mathrm{R}=\mathrm{cL} / 2 \pi \gamma$, where $m$ is the mass of a particle, $\mathrm{N}_{0}$ is the number density of a dust particle (assumed to be a constant), $K$ is the Stoke's resistance coefficient $(=6 \pi \nu \rho \sigma)$ being radius of a dust particle), $v$ is the kinematic viscosity of the fluid.

By introducing the stream functions $\phi(x, y, t), \phi_{p}(x, y, t)$ for the fluid and dust respectively the governing equations (2.2) and (2.3) and the boundary conditions (2.6) and (2.7) become 


$$
\begin{aligned}
& \frac{\partial}{\partial t} \nabla^{2} \phi+\frac{\partial \phi}{\partial y} \nabla^{2} \frac{\partial \phi}{\partial x}-\frac{\partial \phi}{\partial x} \nabla^{2} \frac{\partial \phi}{\partial y}=\frac{1}{R} \nabla^{2} \gamma^{2}+\lambda \alpha \nabla^{2}\left(\phi_{p}-\phi\right), \\
& \frac{\partial}{\partial t} \nabla^{2} \phi_{p}+\frac{\partial \phi_{p}}{\partial y} \nabla^{2} \frac{\partial \phi_{p}}{\partial x}-\frac{\partial \phi_{p}}{\partial x} \nabla^{2} \frac{\partial \phi_{p}}{\partial y}=\alpha \nabla^{2}\left(\phi-\phi_{p}\right), \\
& +\frac{\partial \phi}{\partial y}=0,-\frac{\partial \phi}{\partial x}=\frac{\partial h}{\partial t} \text { at } y=h(x, t), \\
& \left|\frac{\partial \phi}{\partial y}\right|,\left|\frac{\partial \phi}{\partial x}\right|,\left|\frac{\partial p}{\partial y}\right|,\left|\frac{\partial \phi_{p}}{\partial x}\right| \text { all }<\infty \text { as } y \rightarrow \infty .
\end{aligned}
$$

\section{SOLUTION OF THE PROBLEM.}

When Reynolds number becomes larger, the boundary layer is formed. Since we have assumed that the thickness of the boundary layer is larger than the wave amplitude, the regular perturbation technique, which was used for small Reynolds numbers can be applied [1].

If $\delta$ is the thickness of the boundary layer, the non-dimensional variables may be defined as $\tilde{y}=y / \delta$ and $\tilde{\phi}=\phi / \delta, \tilde{\phi}_{p}=\phi_{p} / \delta$. When the viscous term is supposed to be of the same order as the inertia terms, we have that $\delta^{2} R$ is $0(1)$ as usual. The boundary conditions at $y=h$ are expanded into Taylor's series in terms of the inner variables $\tilde{\phi}$ and $\tilde{y}$ as

$$
\begin{aligned}
& \frac{\partial \tilde{\phi}}{\partial x}(0)+\frac{h}{\delta} \frac{\partial^{2} \tilde{\phi}}{\partial x \partial \tilde{y}}(0)+\frac{h^{2}}{2 \delta^{2}} \frac{\partial^{3} \tilde{\phi}}{\partial x \tilde{y}^{2}}(0)+\ldots=-\frac{1}{\delta} \frac{\partial h}{\partial t} \\
& \frac{\partial \tilde{\phi}}{\partial \tilde{y}}(0)+\frac{h}{\delta} \frac{\partial^{2} \tilde{\phi}}{\partial \tilde{y}^{2}}(0)+\frac{h^{2}}{2 \delta^{2}} \frac{\partial^{3} \tilde{\phi}}{\partial \tilde{y}^{3}}(0)+\ldots=0
\end{aligned}
$$

In order that Taylor series converges, $O(\delta)$ must be larger than $0(h)$, that is $0(\varepsilon)<0(\delta)$. Following Tanaka $[1]$, we shall take $\delta=r \varepsilon^{1 / 2}, r$ being an arbitrary constant of $0(1)$, that is $R=\left(r^{2} \varepsilon\right)^{-1}$. The outer flow (the flow beyond the boundary layer) is described by equations (2.8) and (2.9) in terms of the original variables $\left(\phi, \phi_{p}, x, y, t\right)$, while the inner flow (boundary layer flow) is described in terms of the inner variables $\left(\tilde{\phi}, \tilde{\phi}_{p}, x, \tilde{y}, t\right)$, on putting $R=\left(r^{2} \varepsilon\right)^{-1}$ and $\delta=(r \varepsilon)^{1 / 2}$. As $\varepsilon<<1$, we can use perturbation method and assume that

$$
\left(\phi, \phi_{p}, \tilde{\phi}, \tilde{\phi}_{p}\right)=\sum_{n=1}^{\infty} \varepsilon^{n / 2}\left(\phi_{n}, \phi_{p}, \tilde{\phi}_{n}, \tilde{\phi}_{p n}\right)
$$

Substituting (3.3) and using $\tilde{y}=y / \delta, \tilde{\phi}=\phi / \delta, \tilde{\phi}_{\mathrm{p}}=\phi_{\mathrm{p}} / \delta, \mathrm{R}=\left(\mathrm{r}^{2} \varepsilon\right)^{-1}, \quad \delta=(\mathrm{r} \varepsilon)^{1 / 2}$ in the equations $(2.8),(2.9)$ and the boundary conditions (3.1), (3.2) and then equating the coefficients of the like powers of $\varepsilon^{1 / 2}$, we obtain the equations and the boundary conditions corresponding to the first order, second order, etc.

First order $\left(0\left(\varepsilon^{1 / 2}\right)\right)$ 
562

V. RAMAMURTHY AND U.S. RAD

$$
\begin{aligned}
& \text { Outer: } \quad L\left[\phi_{1}\right]=-\alpha \lambda \nabla^{2}\left(\phi_{p 1}-\phi_{1}\right), \\
& L\left[\phi_{p l}\right]=-\alpha \nabla^{2}\left(\phi_{1}-\phi_{p l}\right), \\
& \text { inner: } \quad M\left[\tilde{\phi}_{1}\right]=-\alpha \lambda \frac{\partial^{2}}{\partial \tilde{y}^{2}}\left(\tilde{\phi}_{p l}-\tilde{\phi}_{1}\right), \\
& M_{p}\left[\tilde{\phi}_{p 1}\right]=-\alpha \frac{\partial^{2}}{\partial \tilde{y}^{2}}\left(\tilde{\phi}_{1}-\tilde{\phi}_{p l}\right), \\
& \frac{\partial \tilde{\phi}_{(0)}}{\partial \tilde{y}^{2}}=0, \frac{\partial \tilde{\phi}_{1}}{\partial x}(0)=-\frac{1}{r} \sin (x-t),
\end{aligned}
$$

Second order $O(\varepsilon)$ )

outer: $\quad L\left[\phi_{2}\right]+\alpha \lambda \gamma^{2}\left(\phi_{p 2}-\phi_{2}\right)=\frac{\partial \phi_{1}}{\partial y} \nabla^{2} \frac{\partial \phi_{1}}{\partial x}-\frac{\partial \phi_{1}}{\partial x} \nabla^{2} \frac{\partial \phi_{1}}{\partial y}$

$$
\begin{aligned}
& \mathrm{L}\left[\phi_{\mathrm{p} 2}\right]+\alpha{ }^{2}\left(\phi_{2}-\phi_{\mathrm{p} 2}\right)=\frac{\partial \phi_{\mathrm{p} 1}}{\partial \mathrm{y}} \nabla^{2} \frac{\partial \phi_{\mathrm{pl}}}{\partial \mathrm{x}}-\frac{\partial \phi_{\mathrm{p} 1}}{\partial \mathrm{x}} \nabla^{2} \frac{\partial \phi_{\mathrm{p} 1}}{\partial \mathrm{y}} \\
& \text { inner: } \quad M\left[\tilde{\phi}_{2}\right]+\alpha \lambda \frac{\partial^{2}}{\partial \tilde{y}^{2}}\left(\tilde{\phi}_{p 2}-\tilde{\phi}_{2}\right)=\frac{\partial \tilde{\phi}_{1}}{\partial \tilde{y}} \frac{\partial^{3} \tilde{\phi}_{1}}{\partial x \partial \tilde{y}^{2}}-\frac{\partial \tilde{\phi}_{1}}{\partial x} \frac{\partial^{3} \tilde{\phi}_{1}}{\partial \tilde{y}^{3}} \\
& M_{p}\left[\tilde{\phi}_{p 2}\right]+\alpha \frac{\partial^{2}}{\partial \tilde{y}^{2}}\left(\tilde{\phi}_{2}-\tilde{\phi}_{p 2}\right)=\frac{\partial \tilde{\phi}_{p 1}}{\partial \tilde{y}} \frac{\partial^{3} \tilde{\phi}_{p 1}}{\partial x \partial \tilde{y}^{2}}-\frac{\partial \tilde{\phi}_{p 1}}{\partial x} \frac{\partial^{3} \tilde{\phi}_{p 1}}{\partial \tilde{y}^{3}} \\
& \frac{\partial \tilde{\phi}_{2}}{\partial \tilde{y}}(0)=-\frac{1}{r} \cos (x-t) \frac{\partial^{2} \tilde{\phi}_{1}}{\partial \tilde{y}^{2}}(0), \frac{\partial \tilde{\phi}_{2}}{\partial x}(0)=-\frac{1}{r} \cos (x-t) \frac{\partial^{2} \tilde{\phi}_{1}}{\partial x \partial \tilde{y}}(0), \\
& \text { outer: } \quad \mathrm{L}\left[\phi_{3}\right]+\alpha \lambda \gamma^{2}\left(\phi_{\mathrm{p} 3}-\phi_{3}\right)=-\mathrm{r}^{2} \nabla^{2} \nabla_{\phi_{1}}+\frac{\partial \phi_{1}}{\partial y} \nabla^{2} \frac{\partial \phi_{2}}{\partial x} \\
& +\frac{\partial \phi_{2}}{\partial y} \nabla^{2} \frac{\partial \phi_{1}}{\partial x}-\frac{\partial \phi_{1}}{\partial x} \nabla^{2} \frac{\partial \phi_{2}}{\partial y}-\frac{\partial \phi_{2}}{\partial x} \nabla^{2} \frac{\partial \phi_{1}}{\partial y}
\end{aligned}
$$


inner: $M\left[\tilde{\phi}_{3}\right]+\alpha \lambda\left[r^{2}\left(\frac{\partial^{2} \tilde{\phi}_{p l}}{\partial x^{2}}-\frac{\partial^{2} \tilde{\phi}_{1}}{\partial x^{2}}\right)+\frac{\partial^{2}}{\partial \tilde{y}^{2}}\left(\tilde{\phi}_{p 3}-\tilde{\phi}_{3}\right)\right]=-2 \frac{2}{r^{4} \tilde{\phi}_{1}} \frac{x^{2} \partial \tilde{y}^{2}}{\partial x^{2}}$

$$
+r^{2} \frac{\partial^{3} \tilde{\phi}_{1}}{\partial t \partial x^{2}}+\frac{\partial \tilde{\phi}_{1}}{\partial \tilde{y}} \frac{\partial^{3} \tilde{\phi}_{2}}{\partial x \partial \tilde{y}^{2}}+\frac{\partial \tilde{\phi}_{2}}{\partial \tilde{y}} \frac{\partial^{3} \tilde{\phi}_{1}}{\partial x \partial^{2}}-\frac{\partial \tilde{\phi}_{1}}{\partial x} \frac{\partial^{3} \tilde{\phi}_{2}}{\partial \tilde{y}^{3}}-\frac{\partial \tilde{\phi}_{2}}{\partial x} \frac{\partial^{3} \tilde{\phi}_{1}}{\partial \tilde{y}^{3}},
$$

$M\left[\tilde{\phi}_{\mathrm{p} 3}\right]+\alpha\left[\mathrm{r}^{2} \frac{\partial^{2}}{\partial \mathrm{x}^{2}}\left(\tilde{\phi}_{1}-\tilde{\phi}_{\mathrm{p} 1}\right)+\frac{\partial^{2}}{\partial \tilde{y}^{2}}\left(\tilde{\phi}_{3}-\tilde{\phi}_{\mathrm{p} 3}\right]=\mathrm{r}^{2} \frac{\partial^{3} \phi_{\mathrm{p} 1}}{\partial t \partial \mathrm{x}^{2}}\right.$

$$
+\frac{\partial \tilde{\phi}_{p 1}}{\partial \tilde{y}} \frac{\partial^{3} \tilde{\phi}_{p 2}}{\partial x \partial \tilde{y}^{2}}+\frac{\partial \tilde{\phi}_{p 2}}{\partial \tilde{y}} \frac{\partial^{3} \tilde{\phi}_{p 1}}{\partial x \partial \tilde{y}^{2}}-\frac{\partial \tilde{\phi}_{p 1}}{\partial x} \frac{\partial^{3} \tilde{\phi}_{p 2}}{\partial \tilde{y}^{3}}-\frac{\partial \tilde{\phi}_{p 2}}{\partial x} \frac{\partial^{3} \tilde{\phi}_{p 1}}{\partial \tilde{y}^{3}},
$$

$$
\begin{aligned}
& \frac{\partial \tilde{\phi}_{3}}{\partial \tilde{y}}(0)=-\frac{1}{r} \cos (x-t) \frac{\partial^{2} \tilde{\phi}_{2}}{\partial \tilde{y}^{2}}(0)-\frac{1}{2 r^{2}} \cos ^{2}(x-t) \frac{\partial^{3} \tilde{\phi}_{1}}{\partial \tilde{y}^{3}}(0), \\
& \frac{\partial \tilde{0}_{3}}{\partial x}(0)=-\frac{1}{r} \cos (x-t) \frac{\partial^{2} 0}{\partial x \partial \tilde{y}}(0)-\frac{1}{2 r^{2}} \cos ^{2}(x-t) \frac{\partial^{3} \tilde{o}_{1}}{\partial x \partial \tilde{y}^{2}}(0),
\end{aligned}
$$

Fourth order $\left.\left(0 \varepsilon^{2}\right)\right)$

outer: $L\left[\phi_{4}\right]+\alpha \lambda \nabla^{2}\left(\phi_{p 4}-\phi_{4}\right)=-r^{2} \nabla^{2} \nabla_{\phi_{2}}+\frac{\partial \phi_{1}}{\partial y} \nabla^{2} \frac{\partial \phi_{3}}{\partial x}+\frac{\partial \phi_{2}}{\partial y} \nabla^{2} \frac{\partial \phi_{2}}{\partial x}$

$$
\begin{gathered}
+\frac{\partial \phi_{3}}{\partial y} \nabla^{2} \frac{\partial \phi_{1}}{\partial x}-\frac{\partial \phi_{1}}{\partial x} \nabla^{2} \frac{\partial \phi_{3}}{\partial y}-\frac{\partial \phi_{2}}{\partial x} \nabla^{2} \frac{\partial \phi_{2}}{\partial y}-\frac{\partial \phi_{3}}{\partial x} \nabla^{2} \frac{\partial \phi_{1}}{\partial y}, \\
L\left[\phi_{p 4}\right]+\alpha \nabla^{2}\left(\phi_{4}-\phi_{p 4}\right)=\frac{\partial \phi_{p} 1}{\partial y} \nabla^{2} \frac{\partial \phi_{p} 3}{\partial x}+\frac{\partial \phi_{p} 2}{\partial y} \nabla^{2} \frac{\partial \phi_{p} 2}{\partial x} \\
+\frac{\partial \phi_{p} 3}{\partial y} \nabla^{2} \frac{\partial \phi_{p 1}}{\partial x}-\frac{\partial \phi_{p 1}}{\partial x} \nabla^{2} \frac{\partial \phi_{p} 3}{\partial y}-\frac{\partial \phi_{p 2}}{\partial x} \nabla^{2} \frac{\partial \phi_{p} 2}{\partial y}-\frac{\partial \phi_{p} 3}{\partial x} \nabla^{2} \frac{\partial \phi_{p 1}}{\partial y},
\end{gathered}
$$

inner: $M\left[\tilde{\phi}_{4}\right]+\alpha \lambda\left[r^{2} \frac{\partial^{2}}{\partial x^{2}}\left(\tilde{\phi}_{p 2}-\tilde{\phi}_{2}\right)+\frac{\partial^{2}}{\partial \tilde{y}^{2}}\left(\tilde{\phi}_{p 4}-\tilde{\phi}_{4}\right)\right]=-2 r^{2} \frac{\partial^{4} \tilde{\phi}_{2}}{\partial x^{2} \partial \tilde{y}^{2}}$

$$
\begin{gathered}
+r^{2} \frac{\partial^{3} \tilde{\phi}_{2}}{\partial t \partial x^{2}}+r^{2} \frac{\partial \tilde{\phi}_{1}}{\partial \tilde{y}} \frac{\partial^{3} \tilde{\phi}_{1}}{\partial x^{3}}-r^{2} \frac{\partial \tilde{\phi}_{1}}{\partial x} \frac{\partial^{3} \tilde{\phi}_{1}}{\partial x^{2} \partial \tilde{y}}+\frac{\partial \tilde{\phi}_{1}}{\partial \tilde{y}} \frac{\partial^{3} \tilde{\phi}_{3}}{\partial x \partial \tilde{y}^{2}} \\
+\frac{\partial \tilde{\phi}_{2}}{\partial \tilde{y}} \frac{\partial^{3} \phi_{2}}{\partial x \partial \tilde{y}^{2}}+\frac{\partial \tilde{\phi}_{3}}{\partial \tilde{y}} \frac{\partial^{3} \tilde{\phi}_{1}}{\partial x \partial \tilde{y}^{2}}-\frac{\partial \tilde{\phi}_{1}}{\partial x} \frac{\partial^{3} \tilde{\phi}_{3}}{\partial \tilde{y}^{3}}-\frac{\partial \tilde{\phi}_{2}}{\partial x} \frac{\partial^{3} \tilde{\phi}_{2}}{\partial \tilde{y}^{3}}-\frac{\partial \tilde{\phi}_{3}}{\partial x} \frac{\partial^{3} \tilde{\phi}_{1}}{\partial \tilde{y}^{3}}, \\
M\left[\tilde{\phi}_{p 4}\right]+\alpha\left[r^{2} \frac{\partial^{2}}{\partial x^{2}}\left(\tilde{\phi}_{2}-\tilde{\phi}_{p 2}\right)+\frac{\partial^{2}}{\partial \tilde{y}^{2}}\left(\tilde{\phi}_{4}-\tilde{\phi}_{p 4}\right)\right]=r^{2} \frac{\partial^{3} \tilde{\phi}_{p 2}}{\partial t \partial x^{2}}
\end{gathered}
$$




$$
\begin{aligned}
& +r^{2} \frac{\partial^{1} \phi_{p 1}}{\partial y} \frac{\partial^{3} \phi_{p 1}}{\partial x^{3}}-r^{2} \frac{\partial \tilde{\phi}_{p 1}}{\partial x} \frac{\partial^{3} \tilde{\phi}_{p 1}}{\partial x^{2} \partial \tilde{y}}+\frac{\partial^{3} \tilde{\phi}_{p}}{\partial x \partial \tilde{y}^{2}} \frac{\partial \tilde{\phi}_{p 1}}{\partial \tilde{y}}+\frac{\partial \tilde{\phi}_{p}}{\partial \tilde{y}} \frac{\partial^{3} \tilde{\phi}_{p 2}}{\partial x \partial \tilde{y}^{2}} \\
& +\frac{\partial \tilde{\phi}_{p 3}}{\partial \tilde{y}} \frac{\partial^{3} \tilde{\phi}_{p 1}}{\partial x \partial \tilde{y}^{2}}-\frac{\partial \tilde{\phi}_{p 1}}{\partial x} \frac{\partial^{3} \tilde{\phi}_{p 3}}{\partial \tilde{y}^{3}}-\frac{\partial \tilde{\phi}_{p 2}}{\partial x} \frac{\partial^{3} \tilde{\phi}_{p 1}}{\partial \tilde{y}^{3}}-\frac{\partial \tilde{\phi}_{p 3}}{\partial x} \frac{\partial^{3} \phi_{p 1}}{\partial \tilde{y}^{3}}, \\
& \frac{\partial \tilde{\phi}_{4}}{\partial \tilde{y}}(0)=-\frac{1}{r} \cos (x-t) \frac{\partial^{2} \tilde{\phi}_{3}}{\partial \tilde{y}^{2}}(0)-\frac{1}{2 r^{2}} \cos ^{2}(x-t) \frac{\partial^{3} \tilde{\phi}_{2}}{\partial \hat{y}^{2}}(0) \\
& -\frac{1}{6 r^{3}} \cos ^{3}(x-t) \frac{\partial^{4} \hat{\phi}_{1}}{\partial \tilde{y}^{4}}(0) \text {, } \\
& \frac{\partial \tilde{\phi}_{4}}{\partial x}(0)=-\frac{1}{r} \cos (x-t) \frac{\partial^{2} \tilde{\phi}_{3}}{\partial x \partial \tilde{y}}(0)-\frac{1}{2 r^{2}} \cos ^{2}(x-t) \frac{\partial^{3} \tilde{\phi}_{2}}{\partial x \partial \tilde{y}^{2}}(0) \\
& -\frac{1}{6 r^{3}} \cos ^{3}(x-t) \frac{\partial^{4} \tilde{\phi}_{1}}{\partial x \partial \tilde{y}^{3}}(0) \text {, }
\end{aligned}
$$

and so on.

where

$$
\begin{aligned}
& L(\phi)=-\frac{\partial}{\partial t} \nabla_{\phi}^{2}, L_{p}\left(\phi_{p}\right)=-\frac{\partial}{\partial t} \nabla^{2} \phi_{p}, \\
& M(\tilde{\phi})=\left\{\frac{\partial^{4}}{\partial y^{4}}-\frac{\partial^{3}}{\partial t \partial y^{2}}\right\} \quad \tilde{\phi}, M_{p}\left(\tilde{\phi}_{p}\right)=-\frac{\partial^{3}}{\partial t \partial \tilde{y}^{2}} \tilde{\phi}_{p} .
\end{aligned}
$$

A series of the inner solutions should satisfy the boundary conditions on the wall, while the outer solutions are only restricted to be bounded as y increases, that is

$$
\left|\frac{\partial \phi_{\mathrm{n}}}{\partial \mathrm{x}}\right|,\left|\frac{\partial \phi_{\mathrm{p}}}{\partial \mathrm{x}}\right|,\left|\frac{\partial \phi_{\mathrm{n}}}{\partial \mathrm{y}}\right|,\left|\frac{\partial \phi_{\mathrm{p}}}{\partial \mathrm{y}}\right|<\infty \text { as } \mathrm{y} \rightarrow \infty \text { for } \mathrm{n}=1,2,3,4, \ldots
$$

It is necessary to match the outer and the inner solutions. Following Cole [5] the matching is carried out for both $x$ and $y$ components of the velocity by the following principles:

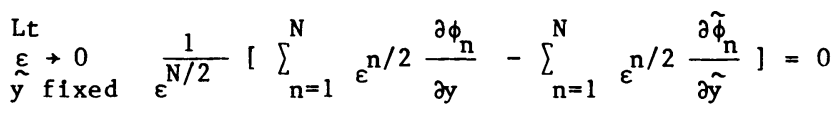

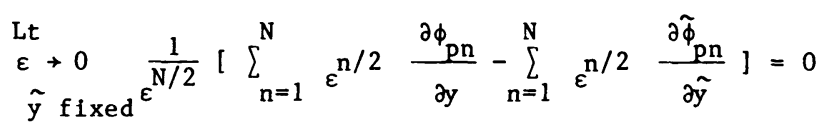

$$
\begin{aligned}
& \underset{\substack{\text { Lt } \\
\tilde{y} \text { fixed }}}{\varepsilon^{N / 2}}\left[\sum_{n=1}^{N} \varepsilon^{n / 2} \frac{\partial \phi_{n}}{\partial x}-r \varepsilon^{1 / 2} \sum_{n=1}^{N} \varepsilon^{n / 2} \frac{\partial \hat{\phi}_{n}}{\partial x}\right]=0
\end{aligned}
$$




$$
\underset{\tilde{y} \text { fixed }}{L t} \frac{1}{\varepsilon^{N / 2}}\left[\sum_{n=1}^{N} \varepsilon^{n / 2} \frac{\partial \phi_{p n}}{\partial x}-r \varepsilon^{1 / 2} \sum_{n=1}^{N} \varepsilon^{n / 2} \frac{\partial \tilde{\phi}_{p n}}{\partial x}\right]=0
$$

up to the $\mathrm{N}$-th order of magnitude

Let us find out first order solutions in the form.

$$
\begin{aligned}
& \tilde{\phi}_{1}(x, \tilde{y}, t)=F_{1}(\tilde{y}) e^{i(x-t)}+F_{1}^{*}(\tilde{y}) e^{-i(x-t)}+F_{1 s}(\tilde{y}), \\
& \tilde{\phi}_{p 1}(x, \tilde{y}, t)=F_{p 1}(\tilde{y}) e^{i(x-t)}+F_{p 1}^{*}(\tilde{y}) e^{-i(x-t)}+F_{p 1 s}(\tilde{y}), \\
& \phi_{1}(x, y, t)=f_{1}(y) e^{i(x-t)}+f_{1}^{*}(y) e^{-i(x-t)}+f_{1 s}(y), \\
& \phi_{p 1}(x, y, t)=f_{p 1}(y) e^{i(x-t)}+f_{p 1}^{*}(y) e^{-i(x-t)}+f_{p 1 s}(y),
\end{aligned}
$$

By substituting ( $3.21 \mathrm{abcd})$ in the first order differential equations ( $3.4 \mathrm{ab)}$ and (3.5ab) and the boundary conditions (3.6) we obtain the following system of equations

$$
\begin{aligned}
& \frac{d^{4} F_{1}}{d \hat{y}^{4}}+1 \frac{d^{2} F_{1}}{d \hat{y}^{2}}=-\alpha \lambda\left[\frac{d^{2} F_{p l}}{d \tilde{y}^{2}}-\frac{d^{2} F_{1}}{d \hat{y}^{2}}\right], \\
& \frac{d^{4} F 1 s}{d y^{4}}=-\alpha \lambda\left[\frac{d^{2} F p l s}{d y^{2}}-\frac{d^{2} F 1 s}{d y^{2}}\right] \text {, } \\
& \frac{d^{2} F p 1}{d \tilde{y}^{2}}=\frac{\alpha}{\alpha-i} \frac{d^{2} F 1}{d \hat{y}^{2}} \\
& \frac{d^{2} F+s}{d \tilde{y}^{2}}=\frac{d^{2} F}{d \tilde{y}^{2}} \\
& \frac{d^{2} f_{1}}{d y^{2}}-f_{1}=0 \\
& \frac{d^{2} f_{p l}}{d y^{2}}-p_{1}=\frac{\alpha}{\alpha-1}\left(\frac{d^{2} f_{1}}{d y^{2}}-f_{1}\right) \\
& \frac{d^{2} f}{d y^{2}}=\frac{d f}{d y^{2}}
\end{aligned}
$$

and their solutions

$$
\begin{aligned}
& F_{1}=D e^{-\lambda \beta \tilde{y}+\frac{1}{2 r}+\lambda_{1} D \beta \tilde{y}-D} \\
& F_{p 1}=\frac{\alpha}{\alpha-1} F_{1}+A \tilde{y}
\end{aligned}
$$




$$
\begin{aligned}
& \frac{d F_{p 1 s}}{d \tilde{y}}=\frac{d F}{d \tilde{y}}=C_{2} \tilde{y}^{2}+C_{3} \tilde{y} \\
& f_{1}=B e^{-y} \\
& f_{p 1}=B_{1} e^{-y}
\end{aligned}
$$

where $\lambda_{1}=\sqrt{-1}(=(1-1) / \sqrt{2}), \quad B=\frac{\left(Q_{1}+1 \alpha \lambda\right)^{1 / 2}}{\sqrt{\alpha^{2}+1}}, Q_{1}=\alpha\left(\lambda^{2}+1\right)+1$ and $D, A$, $C_{1}, C_{2}, B$ and $B_{1}$ are constants. Substituting (3.29) - (3.34) into (3.17) and (3.18), we have

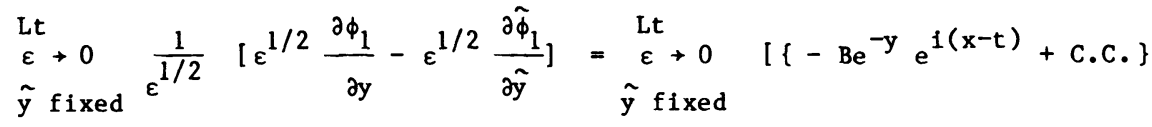

$$
\begin{aligned}
& \left.+C_{1}-\left\{\left(-D \lambda_{1} B e^{-\lambda_{1} \beta \tilde{y}}+D \lambda_{1} \beta\right) e^{i(x-t)}+C . C .\right\}-C_{2} \tilde{y}^{2}-C_{3} \tilde{y}\right]=0
\end{aligned}
$$

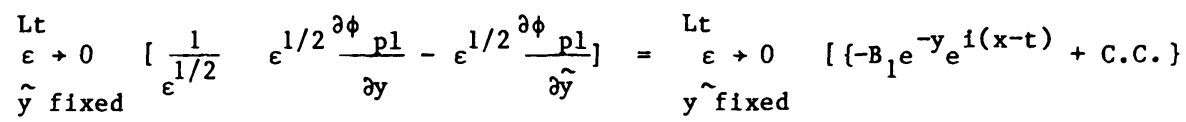

$$
\begin{aligned}
& +C_{1}-\left\{\frac{\alpha}{\alpha-1}-\left(-D \lambda_{1} \beta e^{-\lambda \beta \tilde{y}}+D \lambda_{1} \beta\right)+A e^{i(x-t)}+C . C .\right\} \\
& \left.-c_{2} \tilde{y}^{2}-c_{3} \tilde{y}\right]=0
\end{aligned}
$$

where C.C. stands for the corresponding complex conjugate. Taking into account that $y=r \varepsilon^{1 / 2} \tilde{y}$, we have

$$
e^{-y}=e^{-r} \varepsilon^{1 / 2} \tilde{y}=1-r \varepsilon^{1 / 2} \tilde{y}+\frac{1}{2} r^{2} \varepsilon \tilde{y}^{2}+\ldots(\tilde{y} \text { fixed })
$$

and noting that $\exp \left(-\lambda_{1} \tilde{y}\right)\left(=\exp \left(-\lambda / \mathrm{r} \varepsilon^{1 / 2}\right)\right.$ y, y fixed) decays very rapidly as $\varepsilon \rightarrow 0$ (which is called transcendentally small term (T.S.T) and is neglected in the matching process), we have

$$
\begin{aligned}
& { }_{\varepsilon \rightarrow 0}^{L t}\left[\left(-B+D \lambda_{1} \beta\right) e^{i(x-t)}+C . C .+C_{1}-C_{2} \tilde{y}^{2}-C_{3} \tilde{y}+T \cdot S \cdot T+O\left(\varepsilon^{1 / 2}\right)\right]=0 \\
& \hat{y} \text { fixed } \\
& \underset{\varepsilon \rightarrow 0}{L t}\left[\left(-B_{1}+\frac{D \lambda_{1} \beta \alpha}{\alpha-i}-A\right) e^{I(x-t)}+C \cdot C \cdot+C_{1}-C_{2} \tilde{y}^{2}-C_{3} \tilde{y}+T \cdot S \cdot T+0\left(\varepsilon^{1 / 2}\right]=0\right. \\
& \tilde{y} \text { fixed }
\end{aligned}
$$


Thus the matching condition is satisfied only if

$$
-B+D \lambda_{1} \beta=0,-A-B_{1}+\frac{\alpha}{\alpha-1} D \lambda_{1} \beta=0, C_{1}=C_{2}=C_{3}=0
$$

when similar process is carried out for equations (3.19) and (3.20), we have

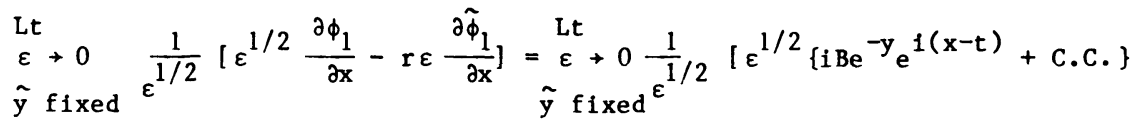

$$
\begin{aligned}
& \left.-r \varepsilon\left\{T . S . T+1 \lambda_{1} D \beta \tilde{y}+1\left(\frac{1}{2 r}-D\right)\right\} e^{i(x-t)}+\text { C.C. }\right]
\end{aligned}
$$

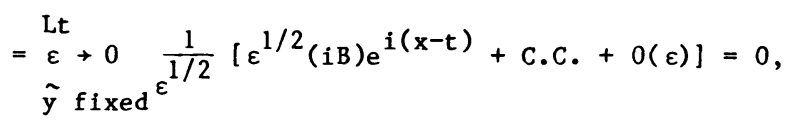

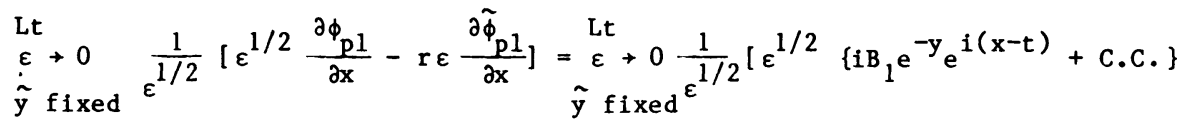$$
\left.-r \varepsilon\left\{T . S . T .+\frac{\alpha}{\alpha-1}\left\{i \lambda_{1} D \beta \tilde{y}+i\left(\frac{1}{2 r}-D\right)\right\}+A \tilde{y}\right\} e^{i(x-t)}+C . C\right]
$$

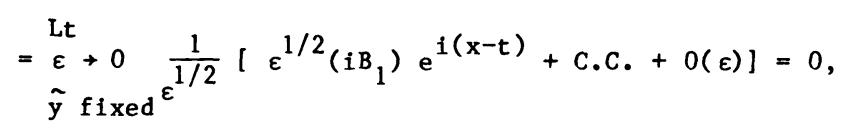

so that the matching condition is satisfied if $B=B_{1}=0$. Thus we have

$$
D=B=B_{1}=A=C_{1}=C_{2}=C_{3}=0
$$

and the first order solutions are obtained as

$$
\begin{aligned}
& \phi_{1}=0, \\
& \phi_{p 1}=0, \\
& \tilde{\phi}_{1}=\frac{1}{2 r} e^{i(x-t)}+\frac{1}{2 r} e^{-i(x-t)} \\
& \tilde{\phi}_{p 1}=\frac{\alpha}{\alpha-i} \frac{1}{2 r}\left[e^{i(x-t)}+e^{-i(x-t)}\right]
\end{aligned}
$$

Next we seek the second order solutions $\tilde{\phi}_{2}, \tilde{\phi}_{\mathrm{p} 2}, \phi_{2}, \phi_{\mathrm{p} 2}$ in the following form

$$
\begin{aligned}
& \tilde{\phi}_{2}=F_{2} e^{2 i(x-t)}+F_{21} e^{i(x-t)}+\text { C.c. }+F_{2 s}, \\
& \tilde{\phi}_{p 2}=F_{p 2} e^{2 i(x-t)}+F_{p 21} e^{i(x-t)}+\text { C.c. }+F_{p 2 s},
\end{aligned}
$$




$$
\begin{aligned}
& \phi_{2}=f_{2} e^{2 i(x-t)}+f_{21} e^{i(x-t)}+c \cdot c \cdot+f_{2 s}, \\
& \phi_{p 2}=f_{p 2} e^{2 i(x-t)}+f_{p 21} e^{i(x-t)}+c \cdot c .+f_{p 2 s},
\end{aligned}
$$

Substituting $(3.44)-(3.47)$ and $(3.48 \mathrm{abcd})$ into $(3.8 \mathrm{ab})-(3.10 \mathrm{ab})$ we get after some calculations

$$
\begin{aligned}
& \tilde{\phi}_{2}=\left(-\frac{i \lambda_{1}}{2 \beta} e^{-\lambda_{1} \beta \tilde{y}}-\frac{1}{2} \tilde{y}+\frac{i \lambda_{1}}{2 \beta}\right) e^{i(x-t)}+\text { c.c. } \\
& \tilde{\phi}_{p 2}=\frac{\alpha}{\alpha-1}\left(-\frac{1 \lambda_{1}}{2 \beta} e^{-\lambda_{1} \beta \tilde{y}}-\frac{1}{2} \tilde{y}+\frac{1 \lambda_{1}}{2 \beta}\right) e^{i(x-t)}+\text { c.c. } \\
& \phi_{2}=\frac{1}{2} e^{-y} e^{i(x-t)}+\text { c.c. } \\
& \phi_{p 2}=\frac{\alpha}{\alpha-i} \frac{1}{2} e^{-y} e^{i(x-t)}+\text { c.c. }
\end{aligned}
$$

Let us now seek third order solutions in the form

$$
\begin{aligned}
& \tilde{\phi}_{3}=F_{3} e^{3 i(x-t)}+F_{32} e^{21(x-t)}+F_{31} e^{i(x-t)}+c . c .+F_{3 s}, \\
& \tilde{\phi}_{p 3}=F_{p 3} e^{3 i(x-t)}+F_{p 32} e^{2 i(x-t)}+F_{p 31} e^{i(x-t)}+c . c .+F_{p 3 s}, \\
& \phi_{3}=f_{3} e^{3 i(x-t)}+f_{32} e^{21(x-t)}+f_{31} e^{i(x-t)}+c . c .+f_{3 s}, \\
& \phi_{p 3}=f_{p 3} e^{3 i(x-t)}+f_{p 32} e^{2 i(x-t)}+f_{p 31} e^{i(x-t)}+c . c .+f_{p 3 s} .
\end{aligned}
$$

where

$$
\begin{aligned}
& F_{3}=0, F_{32}=\frac{1}{4 r} T_{1} e^{-\sqrt{2} \lambda_{1} \tilde{y} \gamma}+\frac{T_{2}}{4 r}-\frac{1}{4 r} T_{3} e^{-\lambda_{1} \tilde{\beta} y}, \\
& F_{31}=-\frac{i r}{2 \beta^{2}} e^{-\lambda_{1} \beta \tilde{y}}+\frac{r}{4} \tilde{y}^{2}-\frac{1 r \lambda_{1}}{2 \beta} \tilde{y}+\frac{1 r}{2 \beta^{2}}, \\
& \frac{d F_{3 s}}{d y}=\frac{Q_{1}}{4 r\left(\alpha^{2}+1\right)}\left[\frac{\lambda_{1}}{\beta} e^{-\lambda_{1} \beta \tilde{y}}+\frac{\lambda_{1}^{*}}{\beta^{*}} e^{-\lambda_{1}^{*} \beta^{*} \tilde{y}}\right]+\frac{a b(a+b)}{\sqrt{2} r\left(a^{2}+b^{2}\right) \sqrt{\alpha^{2}+1}}, \\
& F_{p 3}=0, F_{p 32}=\frac{\alpha}{\alpha-2 i} F_{32}, F_{p 31}=\frac{\alpha}{\alpha-1} F_{31}, \\
& \frac{d F_{p 3 s}}{d \tilde{y}}=\frac{d F_{3 s}}{d \tilde{y}}+\frac{1}{4 r} \frac{\alpha}{\alpha^{2}+1} \lambda_{1} \beta e^{-\lambda_{1} \beta \tilde{y}}-\frac{1}{4 r} \frac{\alpha}{\alpha^{2}+1} \lambda_{1}^{*} \beta^{*} e^{-\lambda_{1}^{*} \beta^{*} \tilde{y}},
\end{aligned}
$$




$$
\begin{aligned}
& \mathrm{f}_{3}=\mathrm{f}_{\mathrm{p} 3}=\mathrm{f}_{32}=\mathrm{f}_{\mathrm{p} 32}=0, \mathrm{f}_{31}=\frac{i \mathrm{r} \lambda_{1}}{2 \beta} e^{-\mathrm{y}}, \mathrm{f}_{\mathrm{p} 31}=\frac{\alpha}{\alpha-1} \frac{\operatorname{ir} \lambda_{1}}{2 \beta} e^{-\mathrm{y}}, \\
& \frac{d f}{d y} s=\frac{d f}{p 3 s}=\frac{a b(a+b)}{d y}= \\
& a=\left(\frac{\left.\sqrt{\left(Q_{1}^{2}+\alpha^{2} \lambda^{2}\right.}\right)+Q_{1}}{2}\right)^{1 / 2}, \quad b=\left(\frac{\left.\sqrt{\left(Q_{1}^{2}+\alpha^{2} \lambda^{2}\right.}\right)-Q_{1}}{2}\right)^{1 / 2} \text {, } \\
& T_{1}=\frac{i \alpha^{2} \lambda \beta}{\sqrt{2} \gamma\left[\alpha^{3}(1+\lambda)-5 \alpha-i\left\{\alpha^{2}(4+\lambda)-2\right\}\right]} \\
& T_{2}=-T_{1}+T_{3}, \\
& \left.T_{3}=\frac{\alpha^{3}(1+\lambda)-5 \alpha-1\left(4 \alpha^{2}-2\right)}{\alpha^{3}(1+\lambda)-5 \alpha-1\left[\alpha^{2}(4+\lambda)-2\right]}, \gamma=\left[1+\frac{\alpha \lambda}{\alpha-2 i}\right]\right]^{1 / 2}
\end{aligned}
$$

We shall now seek the fourth order solutions in the following form

$$
\begin{aligned}
& \tilde{\phi}_{4}=F_{4} e^{4 i(x-t)}+F_{43} e^{3 i(x-t)}+F_{42} e^{2 i(x-t)}+F_{41} e^{i(x-t)}+c \cdot c \cdot+F_{4 s}, \\
& \tilde{\phi}_{p 4}=F_{p 4} e^{4 i(x-t)}+F_{p 43} e^{3 i(x-t)}+F_{p 42} e^{2 i(x-t)}+F_{p 41} e^{i(x-t)}+c \cdot c \cdot+F_{p 4 s}, \\
& \phi_{4}=f_{4} e^{4 i(x-t)}+f_{43} e^{3 i(x-t)}+f_{42} e^{2 i(x-t)}+f_{41} e^{i(x-t)}+c \cdot c \cdot+f_{4 s}, \\
& \phi_{p 4}=f_{p 4} e^{4 i(x-t)}+f_{p 43} e^{3 i(x-t)}+f_{p 42} e^{2 i(x-t)}+f_{p 41} e^{i(x-t)}+c \cdot c \cdot+f_{p 4 s}
\end{aligned}
$$

where

$$
\begin{aligned}
& F_{4}=F_{p 4}=0, \\
& F_{43}=\frac{\lambda_{1}}{48 \delta r^{2}}\left[\frac{2 T_{3} \beta}{3 \delta^{2}-\beta^{2}}\left\{\overline{\sqrt{3}} \beta\left(3 \delta^{2}-\beta^{2}-T_{4}\right)+\delta\left(3 T_{4}-6 \delta^{2}+2 \gamma^{2}\right)\right\}\right. \\
& -\overline{\sqrt{ } 3 \beta^{2}}-\frac{2 \mathrm{~T} 1^{\gamma}}{3 \delta^{2}-2 \gamma^{2}}\left\{2 \sqrt{3 \gamma}\left(3 \delta^{2}-2 \gamma^{2}-\mathrm{T}_{4}\right)+\overline{\sqrt{2} \delta}\left(3 \mathrm{~T}_{4}-6 \delta^{2}+2 \gamma^{2}\right)+\beta \delta\right] \\
& +\frac{\mathrm{T}_{4}}{8 \mathrm{r}^{2}}\left[\frac{\sqrt{2} \lambda_{1} \gamma \mathrm{T}_{1}}{3 \delta^{2}-2 \gamma^{2}} \mathrm{e}^{-\sqrt{2} \lambda_{1} \gamma \tilde{\mathrm{y}}}-\frac{\beta \mathrm{T}_{3} \lambda_{1}}{3 \delta^{2}-\beta^{2}} \mathrm{e}^{-\lambda_{1} \beta \tilde{y}}\right]
\end{aligned}
$$


570

V. RAMAMURTHY AND U.S. RAO

$$
\begin{aligned}
& -\frac{\sqrt{3} \lambda_{1}}{48 \delta r^{2}}\left\{\frac{2 \beta^{2} \mathrm{~T}_{3}\left(3 \delta^{2}-\beta^{2}-\mathrm{T}_{4}\right)}{3 \delta^{2}-\beta^{2}}-\frac{4 \gamma^{2} \mathrm{~T}_{1}\left(3 \delta^{2}-2 \gamma^{2}-\mathrm{T}_{4}\right)}{3 \delta^{2}-2 \gamma^{2}}-\beta^{2}\right\} e^{-\sqrt{3} \lambda_{1} \delta \tilde{y}} \text {, } \\
& F_{p 43}=\frac{\alpha}{\alpha-3 i} F_{43}+\frac{\lambda_{1}^{*} \alpha^{2}}{8 r^{2}\left(\alpha^{2}-3 i \alpha-2\right)(\alpha-3 i)}\left\{\sqrt{2} \gamma \Gamma_{1} e^{-\sqrt{2} \lambda_{1} \tilde{y}_{-}} \mathrm{T}_{3} \beta e^{-\lambda_{1} \beta \tilde{y}}\right\}, \\
& F_{42}=-\frac{T}{2} \tilde{y}+\frac{i \lambda_{1} T_{5}}{4 \beta} \frac{\gamma^{2}}{\left(2 \gamma^{2}-\beta^{2}\right)^{2}}+\frac{T_{5}}{4 \beta\left(2 \gamma^{2}-\beta^{2}\right)^{2}}\left\{\beta\left(2 \gamma^{2}-\beta^{2}\right) \tilde{y}-i \gamma^{2} \lambda_{1}\right\} \\
& e^{-\lambda_{1} \beta \tilde{y}}+\frac{i \lambda_{1}}{2 \sqrt{2} \gamma} \frac{1}{\left(2 \gamma^{2}-\beta^{2}\right)^{2}}\left\{T_{5} \beta^{2}-\left(2 \gamma^{2}-\beta^{2}\right) T_{2}\right\}\left(e^{-\overline{2} \lambda_{1} \gamma \tilde{y}}-1\right), \\
& F_{p 42}=\frac{\alpha}{\alpha-21} F_{42}+\frac{\lambda_{1} e^{-\lambda_{1} \beta \tilde{y}}}{4 \beta(\alpha-1)^{2}(\alpha-2 i)}-\frac{i \alpha^{2} \tilde{y} e^{-\lambda_{1} \beta \tilde{y}}}{4 \beta(\alpha-1)^{2}(\alpha-2 i)}, \\
& F_{41}=\frac{i \lambda_{1}}{\beta}\left[-\frac{1 \gamma^{2}}{2 \beta}+\frac{\lambda_{1}}{2 \beta^{3}} \mathrm{~T}_{8}-\frac{1 \gamma^{2} \mathrm{~T}_{1} \mathrm{~T}_{6}}{4 \mathrm{r}^{2}\left(\beta^{2}-2 \gamma^{2}\right)}+\frac{1 \lambda_{1} \mathrm{~T}_{9}}{\beta^{\star}\left(\beta^{2}+\beta^{\star 2}\right)}+\frac{1}{8 \mathrm{r}^{2}}\left(\beta^{2} \mathrm{~T}_{3}-2 \gamma^{2} \mathrm{~T}_{1}\right)\right. \\
& \left.-\frac{1 \beta^{2}}{8 r^{2}}+\frac{1 \beta^{\star 2}}{16 r^{2}}\right]\left(e^{-\lambda_{1} \beta y^{2}}-1\right)+\frac{\lambda_{1}}{4 \sqrt{2}} \frac{T_{1} T_{6} \gamma}{r^{2}} \frac{1}{\beta^{2}-2 \gamma^{2}}+\frac{T_{9}}{\beta^{\star 2}} \frac{1}{\beta^{2}+\beta^{\star 2}} \\
& -\frac{\lambda_{1}}{4 \mathrm{r}^{2}}\left(-\sqrt{2} \gamma \mathrm{r}_{1}+\beta \mathrm{T}_{3}\right)+\frac{\lambda_{1} \beta}{8 \mathrm{r}^{2}}-\frac{\lambda_{1}^{*} \beta^{*}}{16 \mathrm{r}^{2}}+\frac{\mathrm{T}_{8} \lambda_{1}}{2 \beta^{3}} \tilde{\mathrm{ye}}{ }^{-\lambda_{1} \beta \tilde{y}}-\frac{\lambda_{1}}{4 \sqrt{2}} \frac{\mathrm{T}_{1} \mathrm{~T}_{6} \gamma}{\mathrm{r}^{2}} \frac{\mathrm{e}^{-\sqrt{2} \lambda_{1} \tilde{y}}}{\beta^{2}-2 \gamma^{2}} \\
& -\frac{T_{9}}{\beta^{* 2}} \frac{e^{-\lambda_{1}^{*} \beta^{*} \tilde{y}}}{\beta^{2}+\beta^{* 2}}+\frac{1 r^{2} \lambda 1}{4 \beta} \tilde{y}^{2}-\frac{r^{2} \tilde{y}^{3}}{12}-\frac{1 r^{2}}{2 \beta} \tilde{y}, \\
& F_{p 41}=\frac{\alpha}{\alpha-1} F_{41}-\frac{1 \alpha^{2} \lambda_{1}}{8 r^{2}\left(\alpha^{2}+1\right)(\alpha-1)^{2}}\left[\beta T_{3} e^{-\lambda_{1} \beta \tilde{y}}-\sqrt{2} \gamma_{1} e^{-\sqrt{2} \lambda_{1} \tilde{y}}\right] \\
& +\frac{Q_{1} \alpha}{8 r^{2}\left(\alpha^{2}+1\right)(\alpha-i)^{2}}\left\{1 \lambda_{1} \beta e^{-\lambda{ }_{1} \beta \tilde{y}}+i \lambda_{1}^{*} \beta^{*} e^{-\lambda_{1}^{*} \beta^{*} \tilde{y}}\right\} \\
& -\frac{\lambda_{1}}{8 \mathbf{r}^{2}} \frac{\alpha^{2} \beta e^{-\lambda_{1} \beta \tilde{y}}}{\left(\alpha^{2}+1\right)(\alpha-1)^{2}}+\frac{\lambda_{1}^{*}}{8 r^{2}} \frac{\alpha^{2} \beta^{*} e^{-\lambda_{1}^{*} \beta^{*} \tilde{y}}}{\left(\alpha^{2}+1\right)(\alpha-1)^{2}} \text {, }
\end{aligned}
$$




$$
\begin{aligned}
& \frac{d F_{4 S} s}{d y}=T_{10}\left[\frac{\beta^{2}+\beta^{* 2}}{4 \beta \beta^{*}} \frac{e^{-\left(\lambda_{1} \beta+\lambda_{1}^{*} \beta^{*}\right) \tilde{y}}}{\left(\lambda_{1} \beta+\lambda_{1}^{*} \beta^{*}\right)^{2}}-\frac{e^{-\lambda_{1} \beta \tilde{y}}}{\beta^{2}}\left(\frac{\lambda_{1} \beta}{4} \tilde{y}+\frac{1}{4}+\frac{1 \beta}{4 \beta^{*}}\right)\right. \\
& \left.-\frac{e^{-\lambda_{1}^{*} \beta^{*} \tilde{y}}}{\beta^{* 2}}\left(\frac{\lambda_{1}^{*} \beta^{*} \tilde{y}}{4}+\frac{1}{4}-\frac{1 \beta^{*}}{4 \beta}\right)-\frac{\left(\beta^{2}+\beta^{* 2}\right)}{\beta^{2} \beta^{* 2}}\left(\frac{\beta \beta^{*}}{4\left(\lambda_{1} \beta+\lambda_{1}^{*} \beta^{*}\right)^{2}}-\frac{1}{4}\right)\right],
\end{aligned}
$$

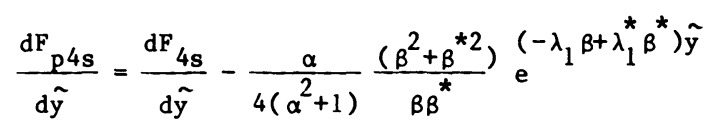

$$
\begin{aligned}
& +\frac{\alpha}{\alpha^{2}+1}\left(\frac{\beta}{4 \beta^{*}}+\frac{1}{4}-\frac{1 \lambda_{1} \beta}{4} \tilde{y}\right) e^{-\lambda \beta_{1} \tilde{y}}+\frac{\alpha}{\alpha^{2}+1}\left(\frac{\beta^{*}}{4 \beta}-\frac{1}{4}+\frac{1 \lambda_{1}^{*} \beta^{*}}{4} \tilde{y}\right) e^{-\lambda_{1}^{*} \beta^{*} \tilde{y}}, \\
& \mathrm{f}_{4}=\mathrm{f}_{\mathrm{p} 4}=\mathrm{f}_{43}=\mathrm{f}_{\mathrm{p} 43}=0, \\
& f_{42}=\frac{T}{4} e^{-2 y}, f_{p 42}=\frac{\alpha r_{2}}{4(\alpha-2 i)} e^{-2 y}, f_{41}=\frac{i r^{2}}{2 \beta^{2}} e^{-y}, \\
& f_{p 41}=\frac{\alpha}{\alpha-1} \frac{i r^{2}}{2 \beta^{2}} e^{-y}, \frac{d f}{d y}=\frac{d f}{d y}=-\frac{T_{10}\left(\beta^{2}+\beta^{* 2}\right)}{\beta^{2} \beta^{\star 2}}\left(\frac{\beta^{*} \beta}{4\left(\lambda_{1} \beta^{*} \lambda_{1}^{*} \beta^{*}\right)^{2}}-\frac{1}{4}\right), \\
& \delta=\left(1+\frac{\alpha \lambda}{\alpha^{-3 i}}\right)^{1 / 2}, \quad T_{4}=\frac{\alpha^{3}(1+\lambda)-11 \alpha+6 i\left(1-\alpha^{2}\right)}{\alpha^{3}-11 \alpha+61\left(1-\alpha^{2}\right)}, \\
& T_{5}=\frac{\alpha^{3}(1+\lambda)-5 \alpha+2 i\left(1-2 \alpha^{2}\right)}{\alpha^{3}-5 \alpha+2 i\left(1-2 \alpha^{2}\right)}, T_{6}=1+\frac{\alpha^{3} \lambda}{(\alpha-2 i)\left(\alpha^{2}+1\right)}, \\
& T_{7}=1+\frac{\alpha^{2} \lambda}{(\alpha-1)^{2}}, T_{8}=-\frac{r^{2} \lambda_{1} \beta}{2}+\frac{\lambda_{1} \beta^{3} T_{3} T_{6}}{8 r^{2}}-\frac{\lambda_{1} \beta_{1} T_{7}}{8 r^{2}\left(\alpha^{2}+1\right)}-\frac{\alpha^{3} \lambda \lambda_{1}^{*} \beta^{3}}{8 r^{2}\left(\alpha^{2}+1\right)(\alpha-1)^{2}}, \\
& \mathrm{~T}_{9}=\lambda_{1}^{*} \beta^{*}\left[\frac{Q_{1} \mathrm{~T}_{7}}{8 \mathrm{r}^{2}\left(\alpha^{2}+1\right)}-\frac{1 \alpha^{3} \beta^{* 2} \lambda}{8 \mathbf{r}^{2}\left(\alpha^{2}+1\right)(\alpha-i)^{2}}\right], \mathrm{T}_{10}=\frac{Q_{1}}{\alpha^{2}+1}
\end{aligned}
$$

In a similar way higher order solutions can also be found. Since it is very laborious to find higher order solutions due to the complexities involved in a dsuty fluid, we are terminating our analysis with a fourth order solution. 


\section{RESULTS AND DISCUSSIONS.}

Thus we found that the third and the fourth solutions consist of the steady part in addition to the periodic one. But the contribution of the steady term in the fourth order solution is more significant to the solution. So we shall take up for discussion the fourth order solution.

The inner steady streaming parts of both the fluid and the dust are plotted against $\tilde{y}$ for various values of the concentration parameter $\lambda$ vide fig. 1 . We find that both in the case of the fluid and the dust the inner steady streaming parts approach to a constant value in the form of the damped oscillation with respect to the distance from the wall.

We see that the progressive motion of the wall causes, at first, the periodic flow in the boundary layer having the same phase as that of the wall motion and then it causes flows of higher harmonics in the boundary layer and induces the periodic flow in the outer layer sucessively. The components of velocities for fluid and dust, both for the outer and the inner flows have been plotted against $y$ and $\tilde{y}$ respectively in figures 2-5, for various values of the parameter $\lambda$ and $(x-t)$, taking $\alpha=2.0$ and Reynolds number $R=500.0$.

We observe from fig. 2 that the axial velocity components $u_{\text {po }}$ of the dust are less than $u_{0}$ of the fluid. It is also seen that while $u_{p o}$ increases as $y$ increases, $u_{o}$ decreases as $y$ increases. The increase in the value of the concentration parameter $\lambda$ results in the increase of the velocity components. But it is interesting to note that both $u_{o}$ and $u_{\text {po }}$ are becoming steady as $y$ increases further and approach almost equal values.

From fig. 3 we observe the nature of the transverse velocity components $v_{0}$ and $v_{\text {po }}$ of the fluid and the particles respectively of the outer flow. The velocity component $v_{\text {po }}$ of the dust is greater than the corresponding value $v_{0}$ of the fluid. Both decrease as $y$ increases and approach more or less the same constant value.

The behaviour of the velocity components $u_{I}$ of the fluid and $u_{p I}$ of the dust of the inner flow can be studied from fig. 4. We note that $u_{p I}$ is greater than $u_{I}$ and $u_{p I}$ are oscillating between positive and negative values.

From fig. 5 we study the nature of the transverse velocity components $v_{I}$ of the fluid and $v_{p I}$ of the dust of the inner flow. We see initially some oscillatory nature in the case of the fluid. But both $v_{I}$ and $v_{p I}$ become steady as $y$ increases.

When $m \rightarrow 0$, the dusty fluid becomes ordinary viscous fluid and then our results are in perfect agreement with those obtained by Tanaka [1] for the case of moderately large Reynolds numbers. 


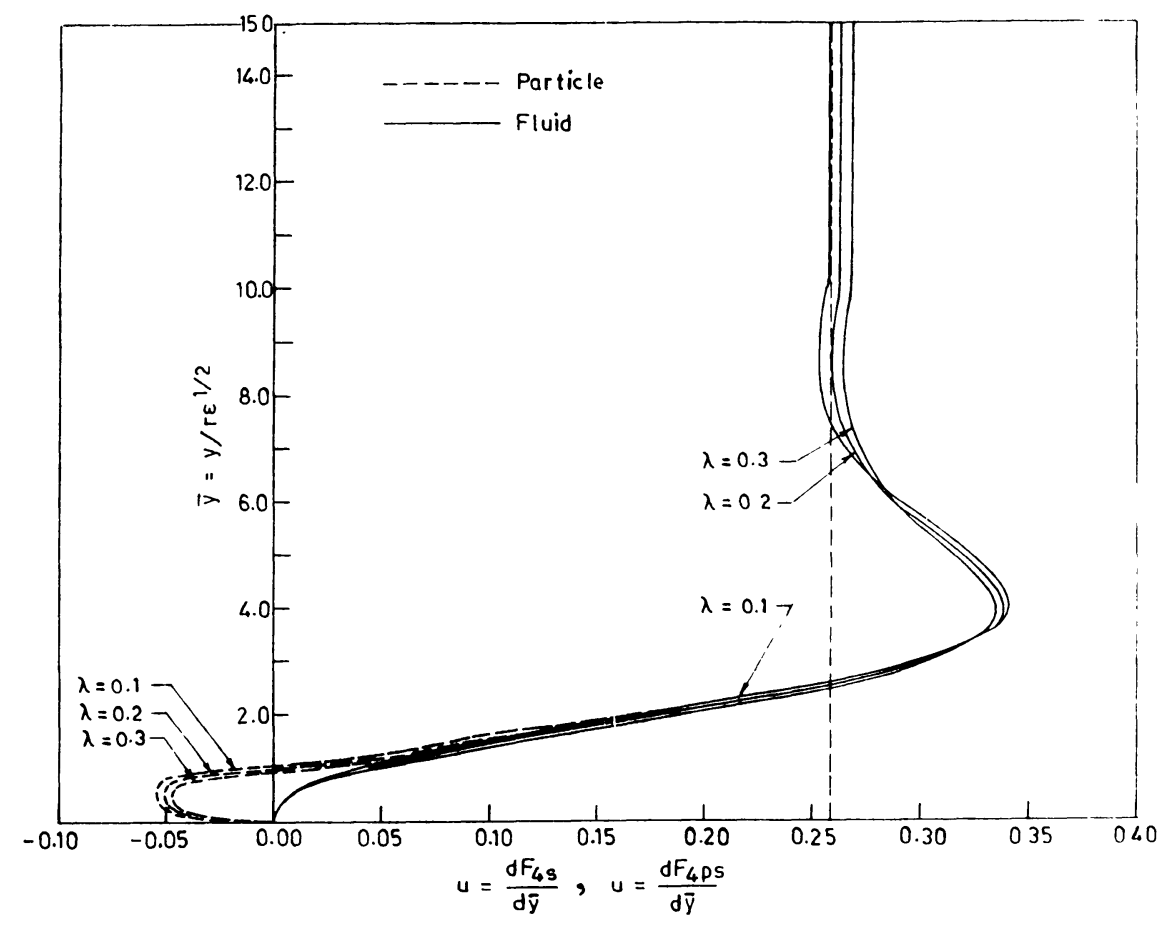

FIG.1 INDUCED STEADY FLOW IN THE BOUNDARY LAYER $\alpha=2.0$

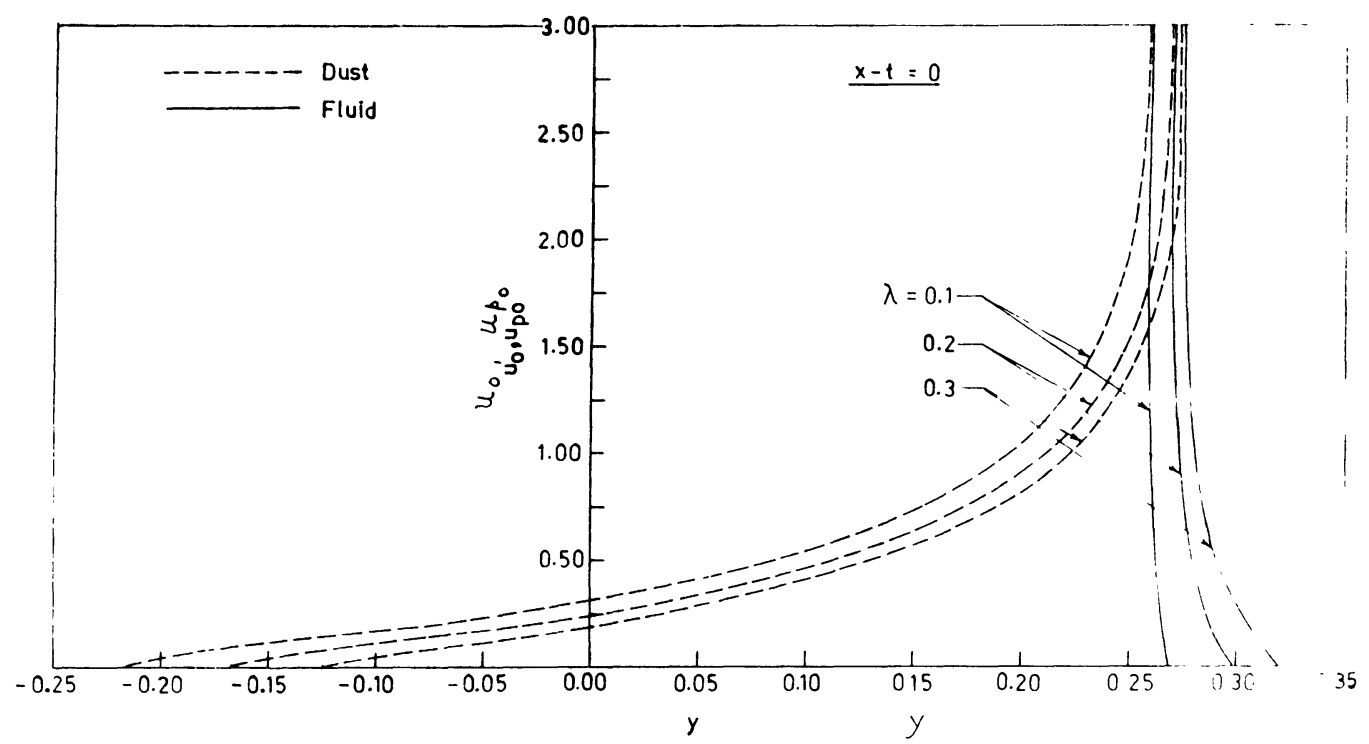

FIG.2 OUTER FLOW AXIAL VELOCITY COMPONENTS OF FLUID UO AND UPO OF PARTICLES FOR R=5r $\alpha=2.0, \varepsilon^{\prime}=0.1$ 


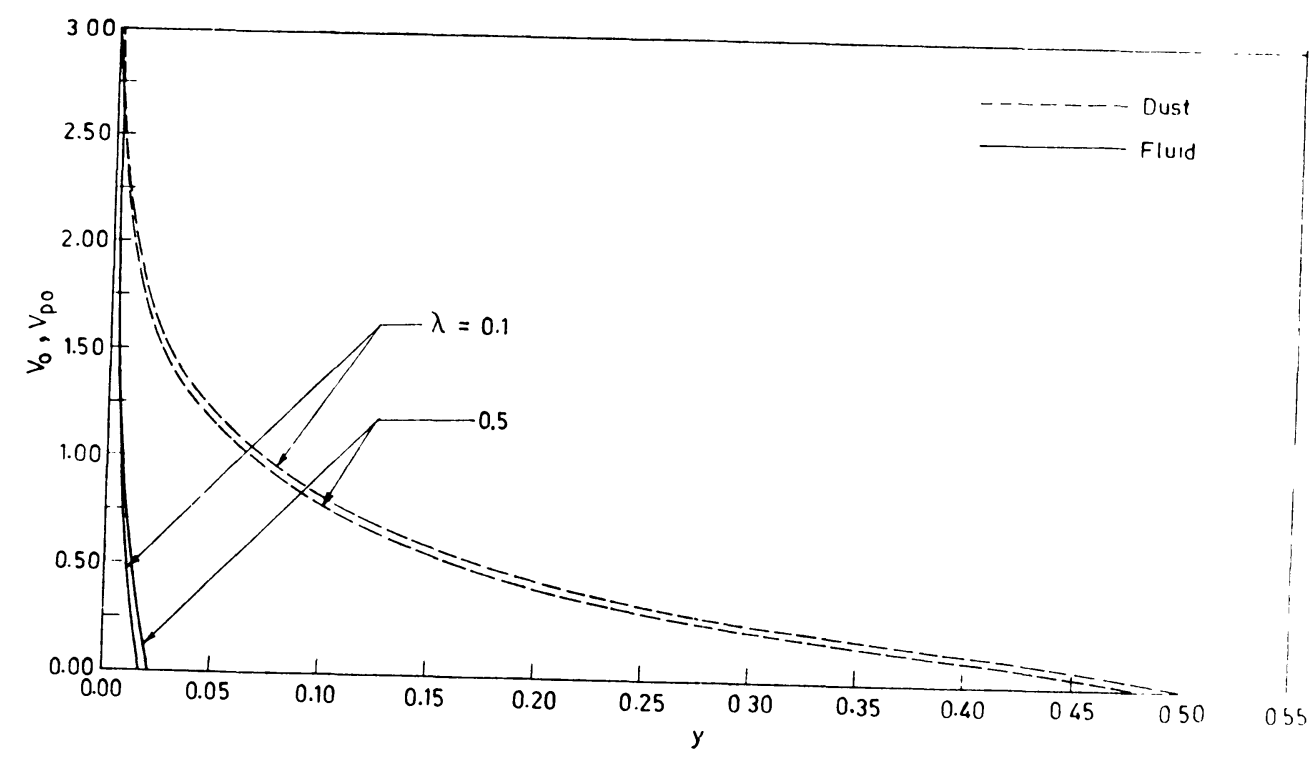

FIG.3 OUTER FLOW TRANSVERSE VELOCITY COMPONENTS OF FLUID VOAND $V_{\text {PO OF PARTICLES FOR }}$ OF
$x-t=0, R=500, \alpha=2.0, \varepsilon=0.1$

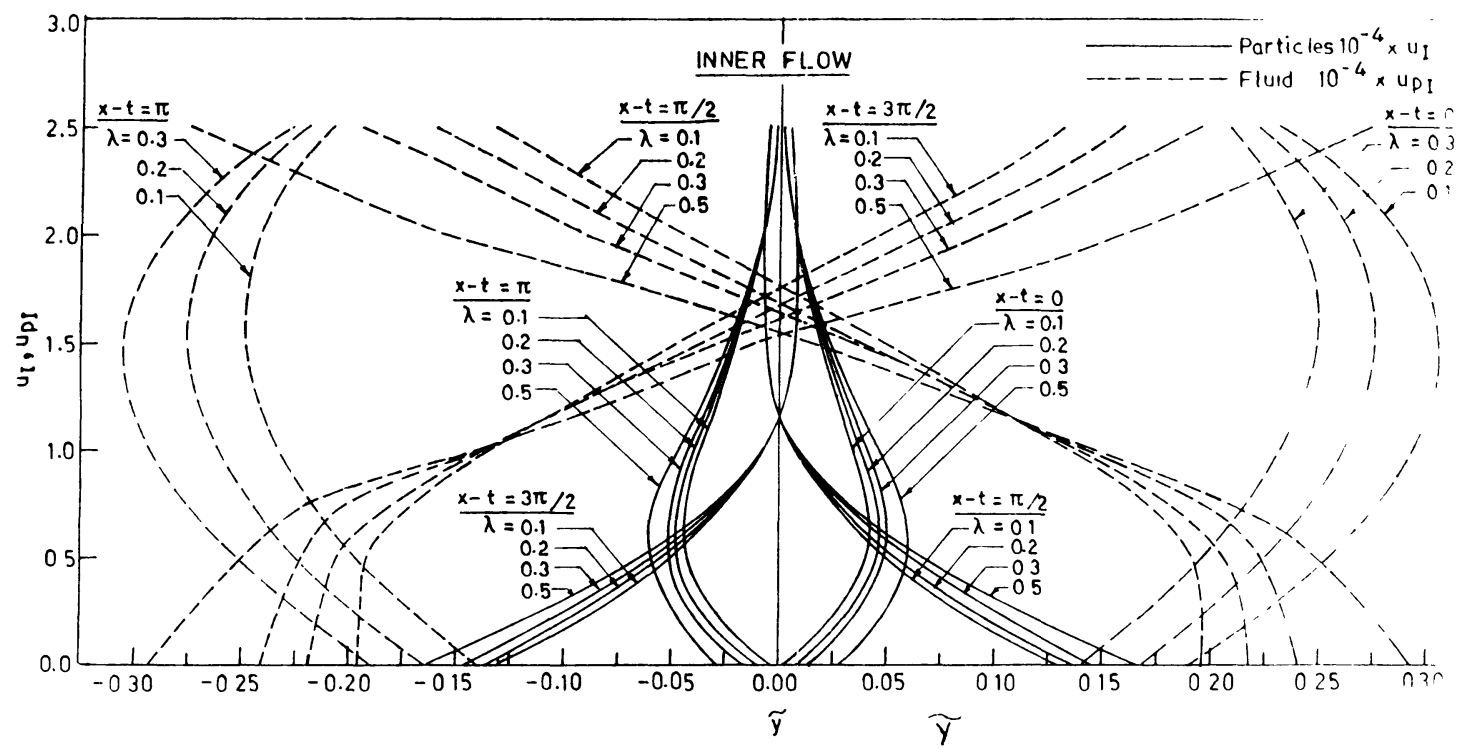

FIG.4 AXIAL VELOCITY COMPONENTS OF FLUID UI AND UPI OF PARTICLES FOR $R=500, \alpha=20, \varepsilon=0.1$, IN THE BOUNDARY LAYER 


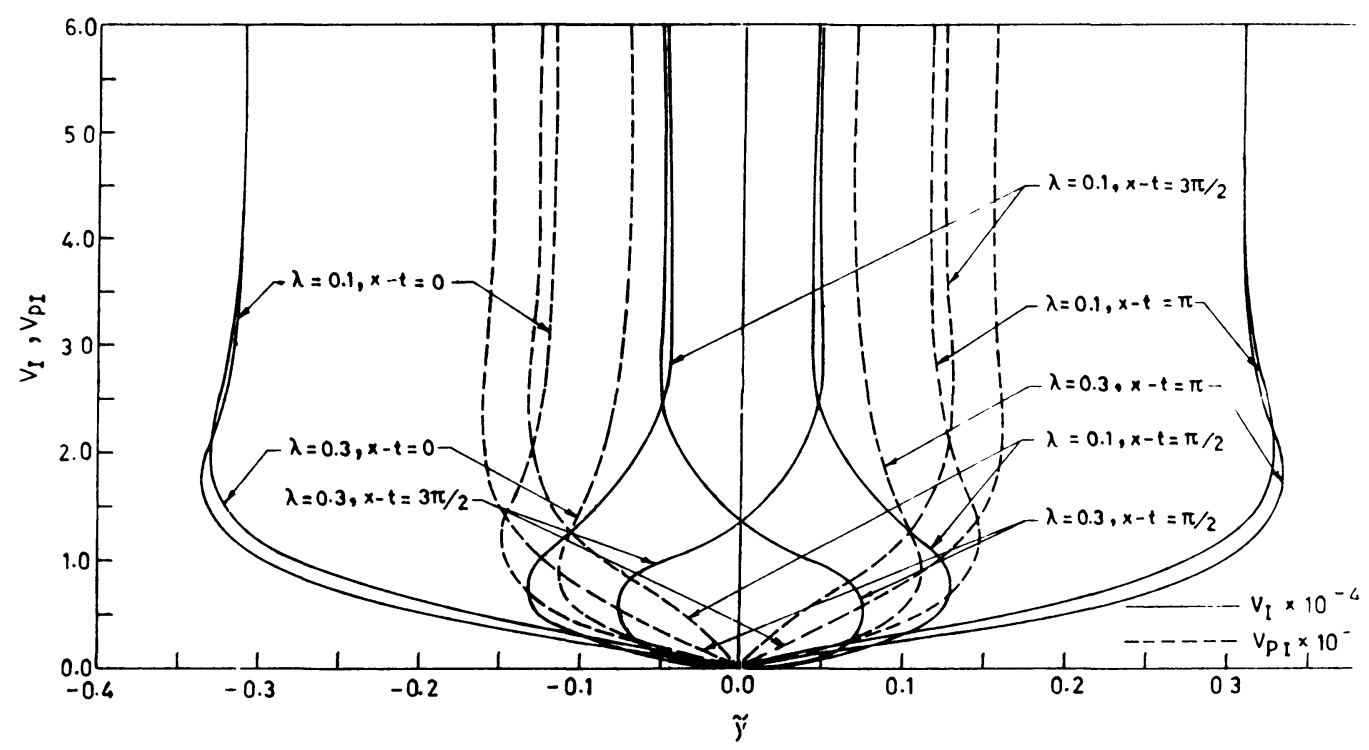

FIG.5 TRANSVESE VELOCTTY COMPONENTS OF FLUID $V_{I}$ ANO $V_{P I}$ OF PARTICLES FOR $R=500, \alpha=2.0$, IN THE BOUNDARY LAYER

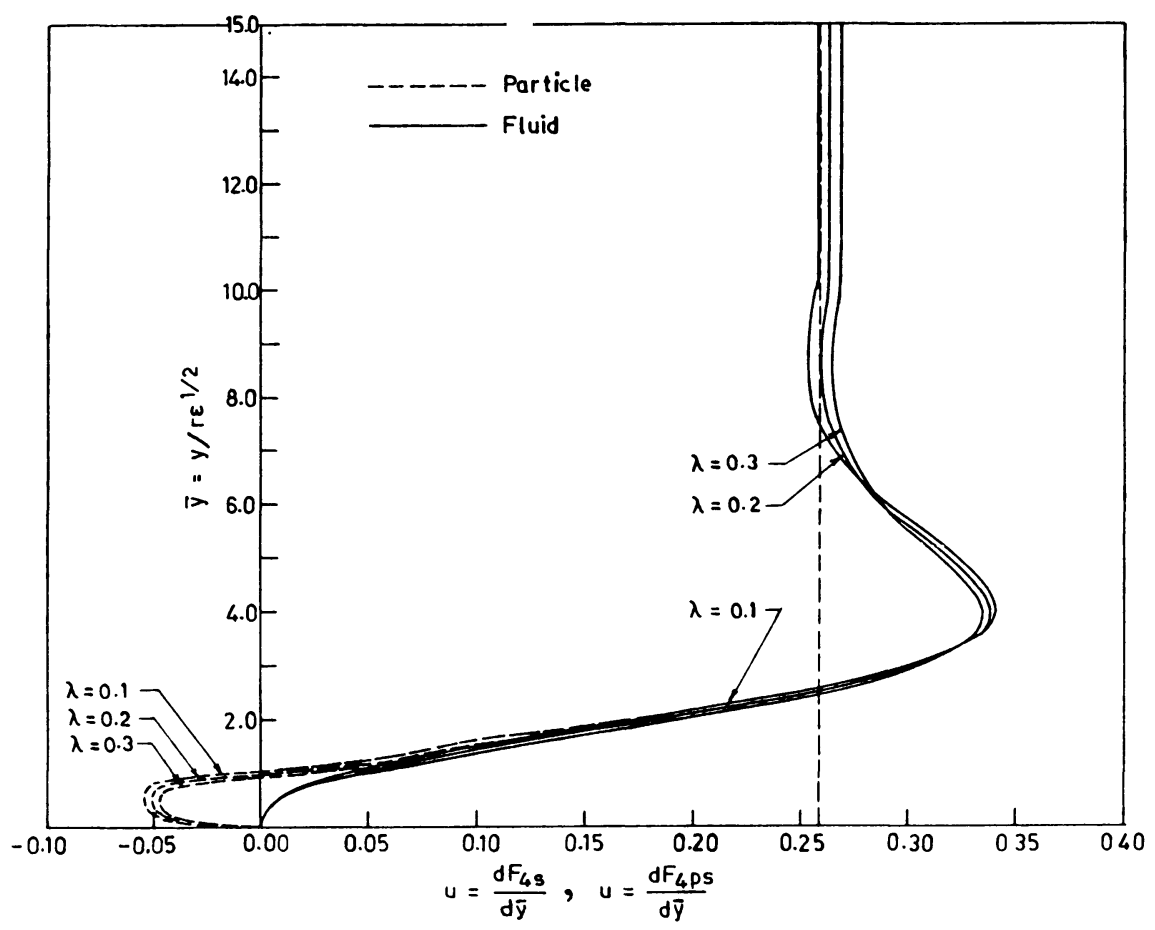

FIG.5.1 INDUCED STEADY FLOW IN THE BOUNDARY LAYER $\alpha=2.0$ 


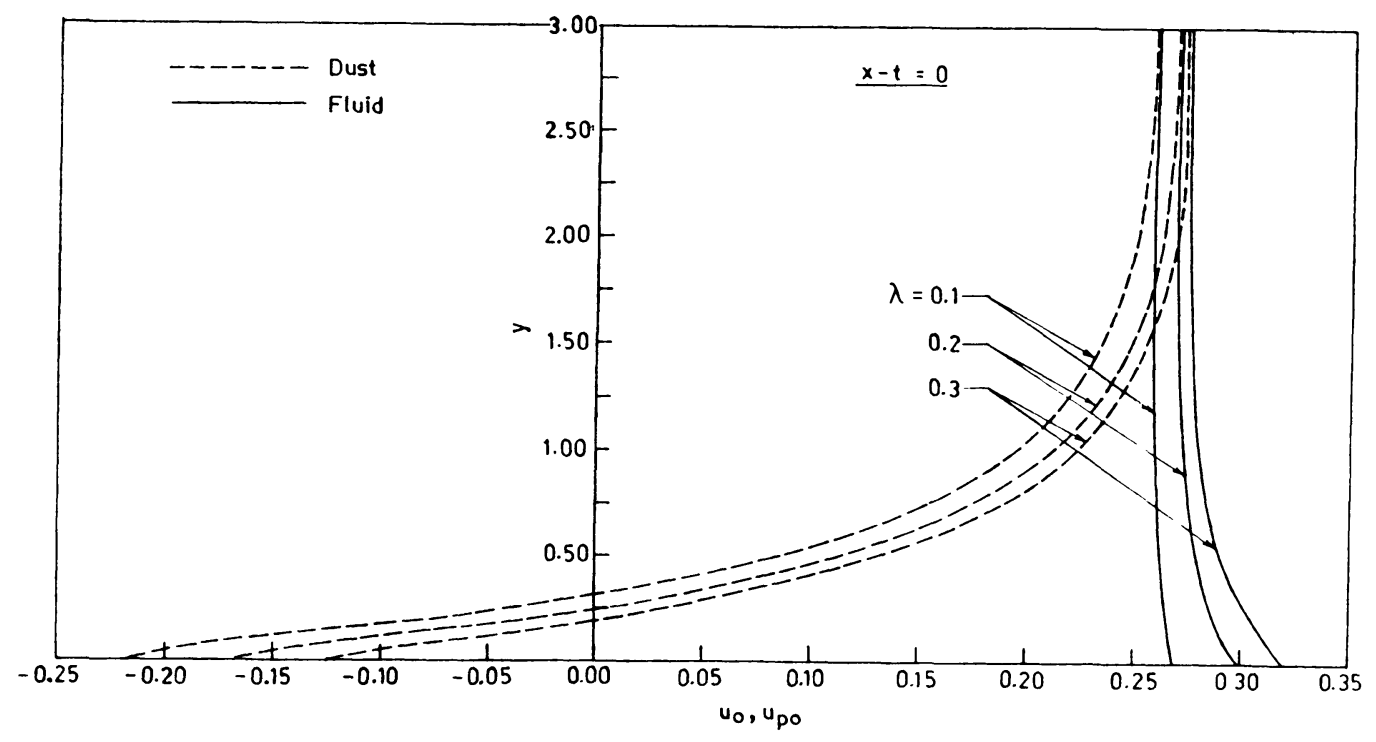

FIG.5.2 OUTER FLOW AXIAL VELOCITY COMPONENTS OF FLUID UO AND UPO OF PARTICLES FOR R=500, $\alpha=2.0, \varepsilon=0.1$

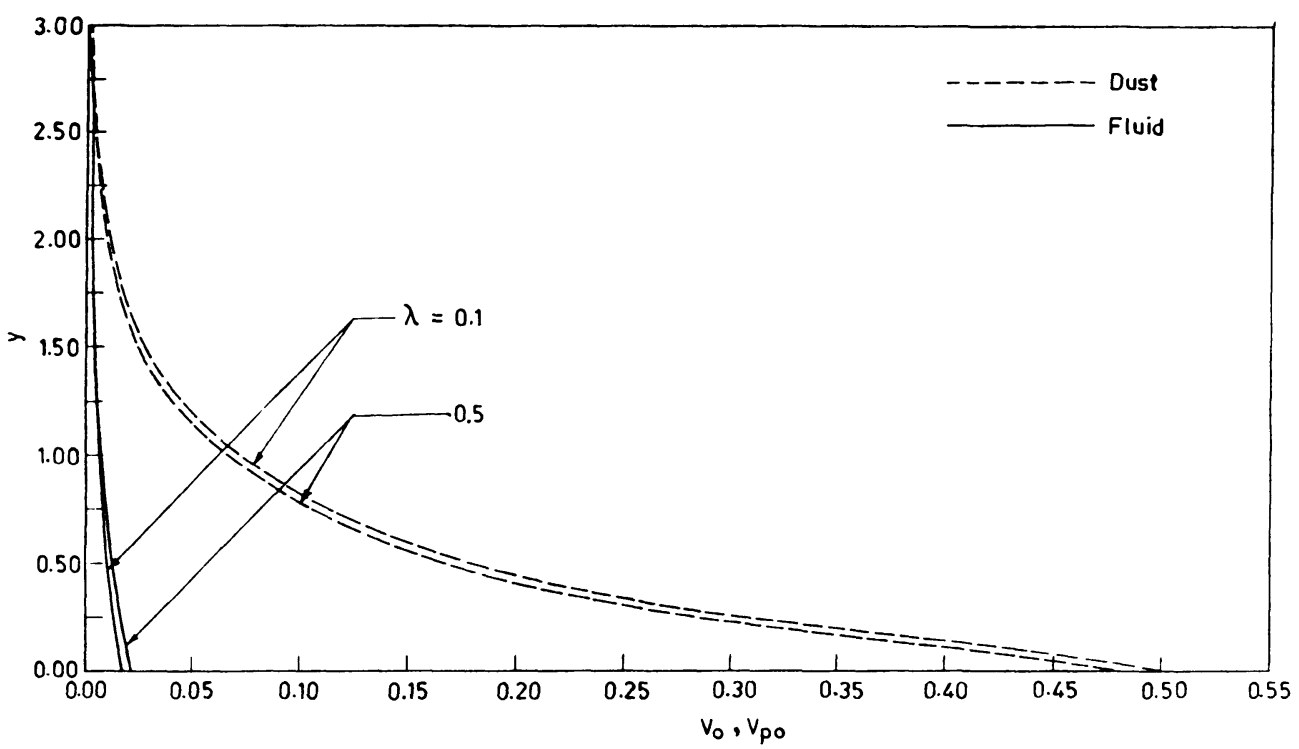

FIG.5.3 OUTER FLOW TRANSVERSE VELOCITY COMPONENTS OF FLUID $V_{0}$ AND $V_{\text {PO }}$ OF PARTICLES FOR $x-t=0, R=500, \alpha=2.0, \varepsilon=0.1$ 


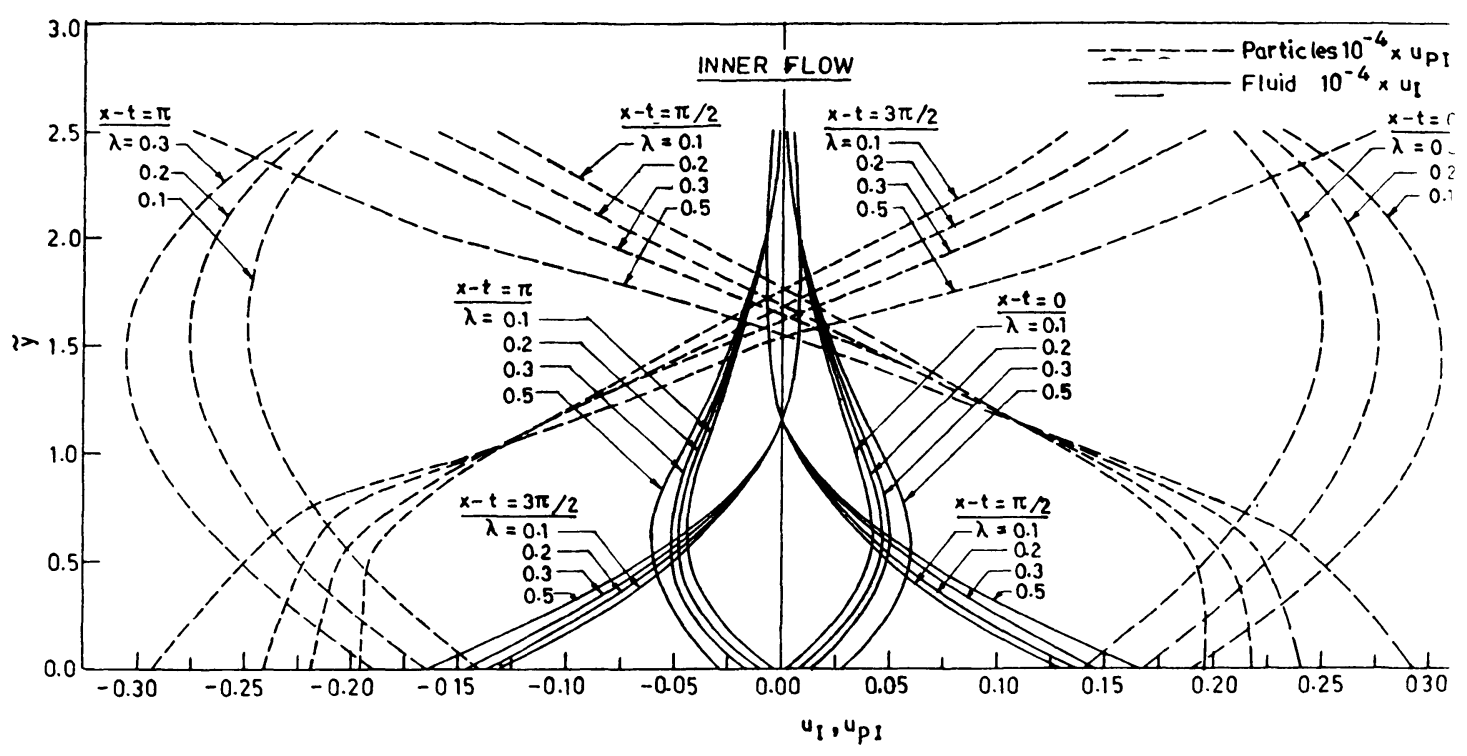

FIG.5.6 AXIAL VELOCITY COMPONENTS OF FLUID UI AND UPI OF PARTICLES FOR $R=500, \alpha=2.0, \varepsilon=0.1$, IN THE BOUNDARY LAYER

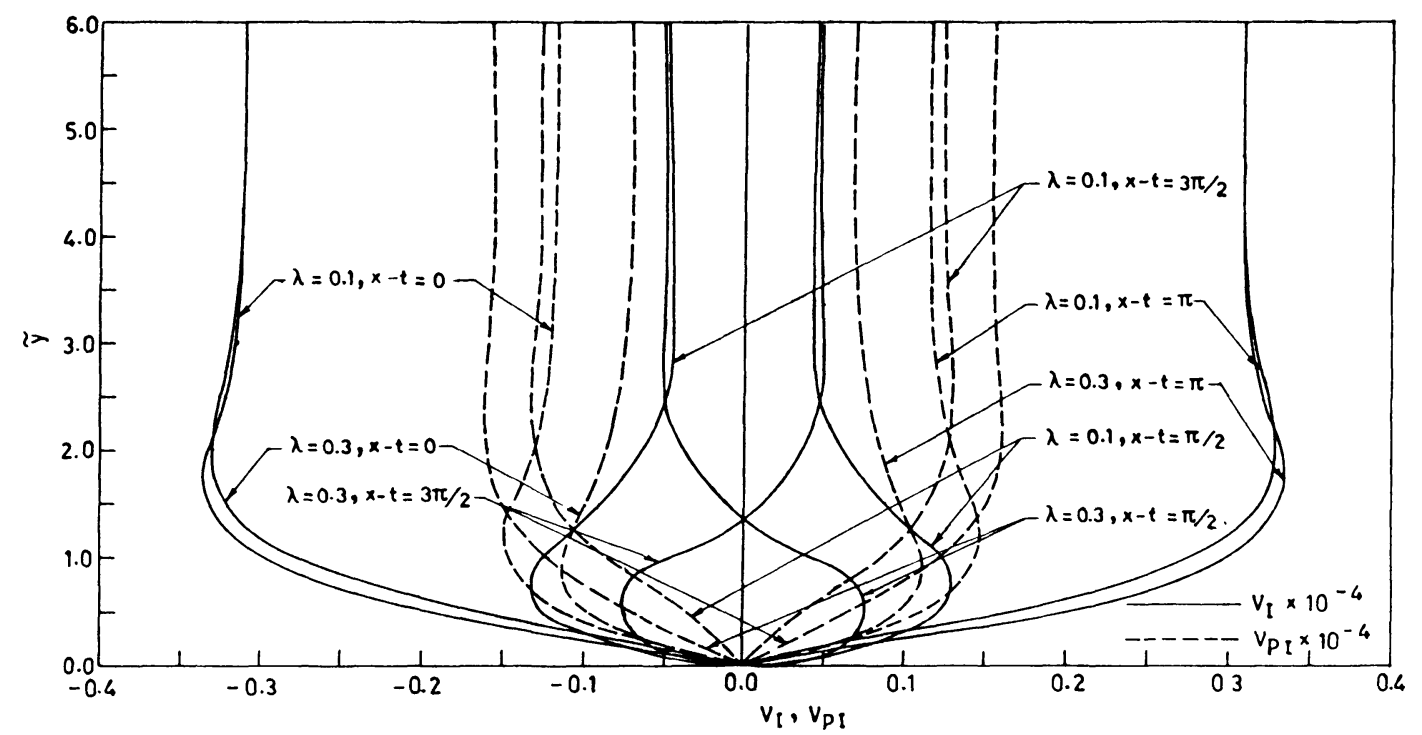

:IG.5.5 TRANSVESE VELOC,ITY COMPONENTS OF FLUID $V_{I}$ AND $V_{P I}$ OF PARTICLES FOR $R=500, \alpha=2.0, \varepsilon=0.1$ IN THE BOUNDARY' LAYER 


\section{REFERENCES}

1. TANAKA, K. Induced Flow due to Wavy Motion of a Wall, J. Phys. Soc. Jpn., 42, 297-305, (1977).

2. TAYLOR, G.I. Analysis of the swimming of microscopic organisms, Proc. Roy. Soc., London A 209,447 (1951).

3. YIN, F.C.P. and FUNG, Y.C. Comparison of theory and experiment in peristaltic transport, J. Fluid Mech. 47,93, (1971).

4. BUMS, J.C. and PARKES, T. Peristaltic motion, J. Fluid, Mech 29, 731, (1967).

5. COLE, J.D. Perturbation Methods in Applied Mathematics (Ginn/Blaisdell, 1968).

6. SAFFMAN, P.G. On the Stability of Laminar flow of a Dusty Gas, J. Fluid Mech, $13,120,(1962)$. 


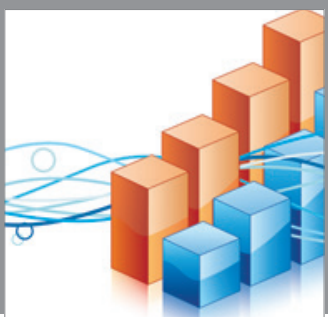

Advances in

Operations Research

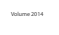

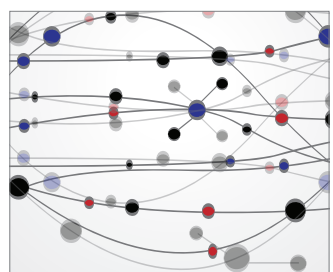

\section{The Scientific} World Journal
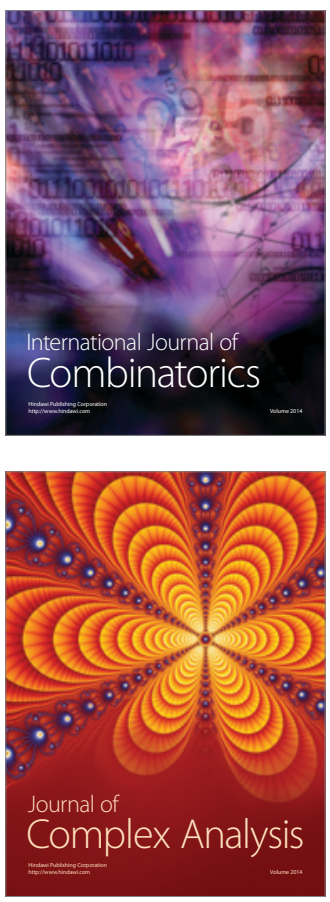

International Journal of

Mathematics and

Mathematical

Sciences
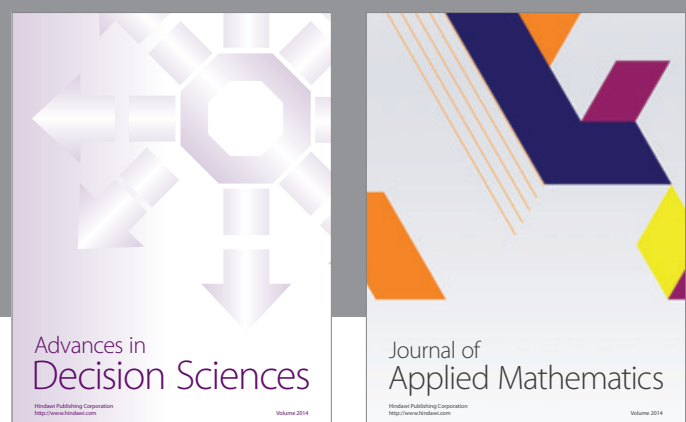

Journal of

Applied Mathematics
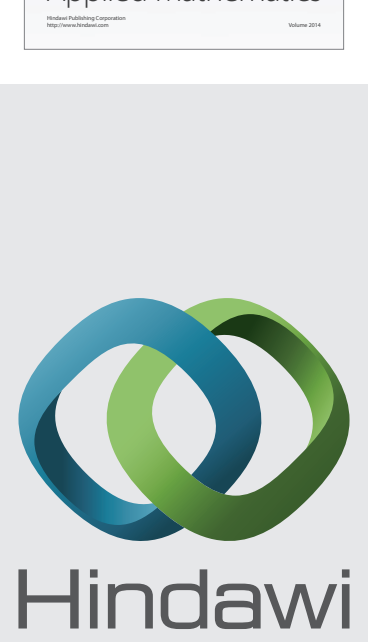

Submit your manuscripts at http://www.hindawi.com
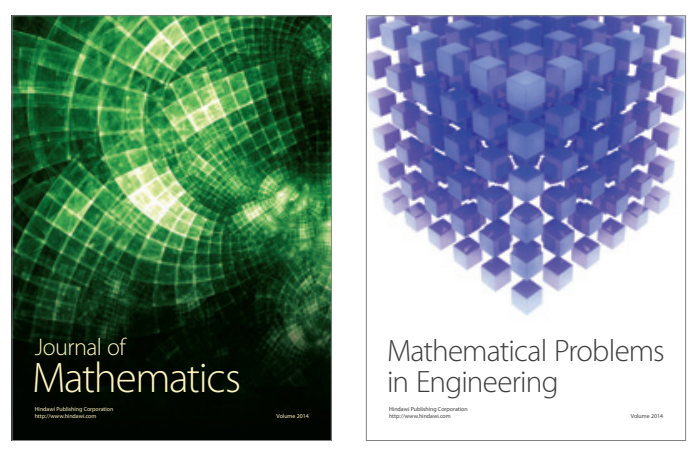

Mathematical Problems in Engineering
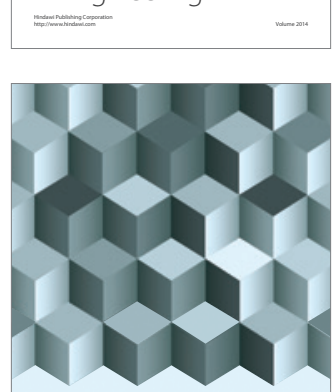

Journal of

Function Spaces
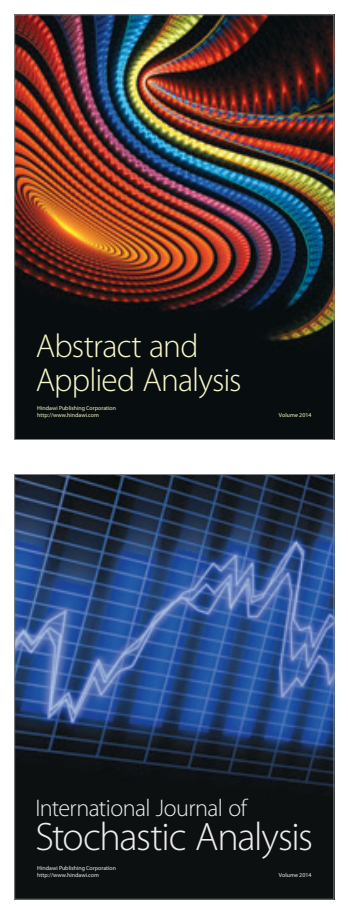

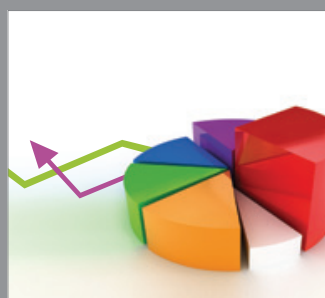

ournal of

Probability and Statistics

Promensencen
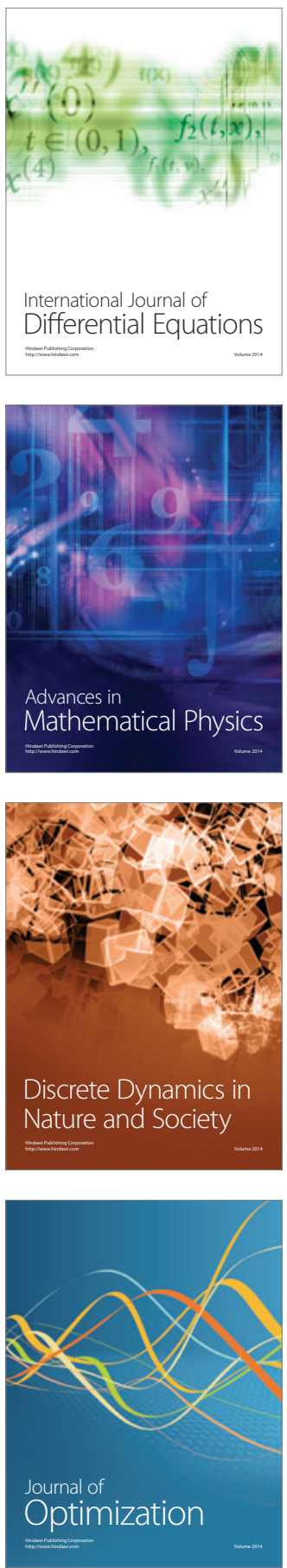